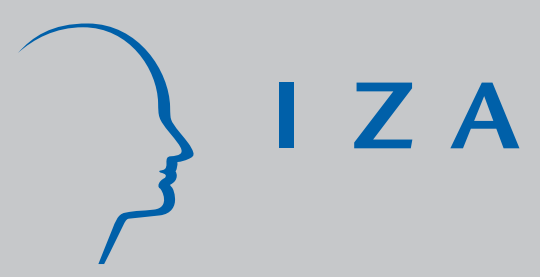

IZA DP No. 2549

Technological and Organizational Changes, and Labor Flows: Evidence on French Establishments

Philippe Askenazy

Eva Moreno-Galbis

J anuary 2007 


\title{
Technological and Organizational Changes, and Labor Flows: Evidence on French Establishments
}

\author{
Philippe Askenazy \\ Paris Sciences Economiques \\ and IZA
}

Eva Moreno-Galbis

Université du Mans

Discussion Paper No. 2549

January 2007

IZA

P.O. Box 7240

53072 Bonn

Germany

Phone: +49-228-3894-0

Fax: +49-228-3894-180

E-mail: iza@iza.org

Any opinions expressed here are those of the author(s) and not those of the institute. Research disseminated by IZA may include views on policy, but the institute itself takes no institutional policy positions.

The Institute for the Study of Labor (IZA) in Bonn is a local and virtual international research center and a place of communication between science, politics and business. IZA is an independent nonprofit company supported by Deutsche Post World Net. The center is associated with the University of Bonn and offers a stimulating research environment through its research networks, research support, and visitors and doctoral programs. IZA engages in (i) original and internationally competitive research in all fields of labor economics, (ii) development of policy concepts, and (iii) dissemination of research results and concepts to the interested public.

IZA Discussion Papers often represent preliminary work and are circulated to encourage discussion. Citation of such a paper should account for its provisional character. A revised version may be available directly from the author. 
IZA Discussion Paper No. 2549

January 2007

\section{ABSTRACT}

\section{Technological and Organizational Changes, and Labor Flows: Evidence on French Establishments}

This paper investigates the effects of organizational and technological changes on job stability of different occupational categories in France. We conduct an empirical analysis in which we make extensive use of a unique data set on a representative sample of French establishments. Working with various indicators of labor flows (gross labor flows, hiring rate, firing rate, net labor flows and churning flows), we find that the use of new technology seems to have a positive effect on aggregate job turnover and, more specifically, turnover among manual workers. In contrast, innovative workplace organizational practices are related to lower turnover among clerical workers and intermediate professionals and have a positive effect on churning among managers.

JEL Classification: J23, J41, J63, L23, O33

Keywords: labor flows, information and communication technologies, organizational change

Corresponding author:

Philippe Askenazy

Paris Sciences Economiques

$48 \mathrm{Bd}$ Jourdan

75014 Paris

France

E-mail: askenazy@pse.ens.fr 


\section{Introduction}

The consequences of the information and communication technologies (ICT) revolution have been widely analyzed in the economic literature. Nowadays, there is widespread agreement among economists as to the skilled labor and high performance workplace organizational practices (see Machin and Van Reenen (1998), Krusell et al. (2000) or Bresnahan, Brynjolfsson, and Hitt (2002)).

In spite of the extensive literature on the effects of the ICT revolution, there is not much evidence about its consequences for job quality. The aim of this paper is to provide an empirical contribution to the small, but growing literature on this topic. Table 1 reflects the fact that the introduction of ICTs and high performance workplace organizational practices (HPWOPs), such as delayering ${ }^{1}$, team work, decentralization of decision-making within firms and quality control procedures, is concomitant, in most European countries, with an increased feeling of job instability among workers. This feeling contrasts with the observed global job instability, which has risen only slightly (see Givord and Maurin (2004) for France). How can this paradox be explained? We suggest that the apparently neutral impact of new technologies and HPWOPs hides significant heterogeneities among workers. In other words, some categories of workers have seen their job stability negatively affected by new technological and organizational practices, while others have been positively affected. These heterogeneous effects promote a feeling of instability among workers, even though aggregate job stability has remained roughly constant.

Within the extensive literature on job flows ${ }^{2}$, some recent papers offer evidence that partially supports this assertion. Michelacci and Lopez-Salido (2004) develop a theoretical approach in which they find that, while neutral technology shocks increase job creation and job destruction (reducing aggregate employment), investment-specific shocks increase job destruction and have mild effects on job creation (aggregate employment expands). On the empirical side, Bauer and Bender (2004), working with a matched employer-employee panel data set relating to Germany,

\footnotetext{
${ }^{1}$ Delegating responsibilities to lower hierarchical levels inside the establishment by removing one or more managerial levels.

${ }^{2}$ See for example Burgess and Nickell (1990) for the UK, Hamermesh, Hassink, and Van-Ours (1996) for the Netherlands, Burgess, Lane, and Stevens (2000) or Neumark, Polsky, and Hansen (1999) for the US, Ilmakunnas and Maliranta (2003) for Finland and Abowd and Kramarz (2003) for France.
} 


\begin{tabular}{|c|c|c|c|c|c|c|c|}
\hline & \multicolumn{2}{|c|}{$\begin{array}{l}\text { ICT }^{1} \text { investment } \\
\text { in OECD countries }\end{array}$} & \multicolumn{4}{|c|}{ HPWO $^{2}$ practices } & $\begin{array}{l}\text { Evolution }^{3} \text { of } \\
\text { job stability }\end{array}$ \\
\hline & 1980 & 2000 & $\begin{array}{l}\text { Task } \\
\text { rotation }\end{array}$ & $\begin{array}{c}\text { Working } \\
\text { teams }\end{array}$ & $\begin{array}{c}\text { Higher } \\
\text { worker implication }\end{array}$ & $\begin{array}{l}\text { Reduction } \\
\text { in hierarchy }\end{array}$ & 1985-1995 \\
\hline France & 6.1 & 13.1 & 6 & 30 & 44 & 21 & $-14^{*}$ \\
\hline Germany & 7.7 & 19.2 & 7 & 20 & 19 & 30 & $-18^{*}$ \\
\hline Italy & 8.0 & 16.7 & 13 & 28 & 24 & 10 & $-5^{*}$ \\
\hline United Kingdom & 5.6 & 22.0 & 13 & 33 & 48 & 45 & $-22^{*}$ \\
\hline
\end{tabular}

1. Percentage of non residual gross fixed capital formation, total economy. ICT equipment is defined as computer and office equipment and communication equipment; software includes both purchased and own account software.

2. Percentage of establishments stating in 1996 some of the HPWO practices adopted by their employers during the three previous years (concerning Italy data refers to the three previous months).

3. Evolution in percentage points in the proportion of workers considering their job ensured.

.. Unavailable data.

Source concerning ICT: OECD estimates based on national accounts.

Source concerning HPWO: OECD Employment Outlook 1999, table 4.4, page 206

Source concerning job stability: OECD Employment Outlook 1997, table 5.2, page 148, and table 5.3, page 149.

Table 1: ICT investment, HPWO practices and job stability in some OECD countries.

conclude that organizational changes are skill-biased since they lead to higher job destruction and separation rates for low and medium-skill workers, while the employment patterns of highskill workers are not significantly affected. They also find that new technologies do not have significant effects on gross job and worker flows. Greenan and Guellec (2000), using a sample of French firms over the period 1986-1990, show that innovative firms create more jobs than others but that job creation in non-innovative firms is reduced. Neumark and Reed (2004), working with US data, estimate a positive link between new economy jobs and contingent ${ }^{3}$ or non-standard ${ }^{4}$ employment relationships. Finally, Givord and Maurin (2004), using the French Labor Force Survey, develop an econometric analysis in an attempt to identify the structural factors that have driven the increase in the risk of involuntary job loss experienced by French

\footnotetext{
${ }^{3} \mathrm{~A}$ contingent worker is defined as an individual holding a job that is temporary by its nature.

${ }^{4}$ Non-standard employment arrangements are: independent contractors, on-call workers, temporary agency workers and workers provided by contract firms.
} 
workers over the last 20 years. They conclude that technological change is the main factor responsible for the increased job insecurity, but that its effect may be mitigated by institutional changes.

Whereas most of the existing literature on the topic focuses on the effects of ICTs and HPWOPs on aggregate labor flows or on the labor flows of skilled and unskilled workers, our paper analyzes the impact of innovative technological and organizational practices on the labor flows (gross labor flows, hirings, firings, net labor flows and churning flows) of various occupational categories (managers, intermediate professions, white-collar workers and manual workers). We use a cross-sectional database resulting from the merging of three French surveys conducted in 1999 and covering more than 2,500 establishments. These surveys are 1) the REPONSE survey (RElations PrOfessionnelles et NégociationS d'Entreprise), which describes establishments use of new technologies and innovative organizational practices, 2) the DMMO survey (Déclaration Mensuelle de Mouvements de main d'Oeuvre), which describes establishments gross labor and job flows (entries, exits, jobs created and lost, etc.) by gender, age, occupational category, etc., and 3) the EMMO survey (Enquête sur les Mouvements de Main d'Oeuvre), a quarterly survey describing entries and exits from employment. Unfortunately, whether we consider flow variables (labor flows) or stock variables (the use of ICTs and HPWO practices), we currently have detailed information for only one year, 1998, which obliges us to adopt a cross-sectional approach.

We find that ICTs are positively related to turnover among blue- collar workers, whereas many HPWO practices transferring "power" to production workers (such as delayering or autonomous work and project groups) are associated with higher churning flows among white-collar workers and are negatively related to turnover among clerical workers and intermediate professions. Correlations with net employment change are also significant and consistent with biased practices, but their magnitude is small compared to the coefficient for hirings plus exits. Our estimations suggest that biased technological and organizational shocks increase labor flows in the occupational category whose relative productivity is damaged by the shock.

The paper is organized as follows. Section 2 describes both the data surveys and the data itself. Section 3 details the econometric analysis developed in the paper. The results are explained in 
section 4 , while some conclusions are drawn in section 5 .

\section{The data}

The database we use results from the merging of three French surveys conducted in 1999 but referring to 1998: the REPONSE survey ${ }^{5}$ (RElations PrOfessionnelles et NégociationS d'Entreprise), the DMMO survey (Déclaration Mensuelle de Mouvements de main d'Oeuvre) and the EMMO survey (Enquête des Mouvements de Main d'Oeuvre). The database is collected by the French Ministry of Labor. We use these data under the terms of an agreement with the Ministry.

In REPONSE, more than 2,500 establishments were surveyed, with senior managers being asked about the economic situation of the establishment, its internal organization, technological changes, wage bargaining with unions and disputes with workers. Only establishments with 20 or more employees were sampled and no public-sector employees were included (except workers in state-owned industries). With regard to ICTs and HPWO practices, managers were asked about their presence in the establishment and, in some cases, about the proportion of workers using the corresponding technology or workplace practice. REPONSE, together with the COI Survey (Changement Organisationnel et Informatisation), is the main source of data on French firms organizational and technological choices.

In the DMMO survey, each establishment with at least 50 employees makes a monthly declaration of employment at the beginning and end of each month and the total entries and exits over the course of the month. Respondent establishments also report the nature of all employment transactions (type of contract offered to new entries and reasons for exits), as well as the skill level, age, seniority, occupation, etc. of employees involved in the transactions.

The EMMO survey is a quarterly survey covering establishments with 10 to 49 employees, excluding publicly-owned establishments and domestic services. It provides information on the number of entries and exits from employment.

\footnotetext{
${ }^{5}$ The REPONSE survey was also conducted in 1993, but only a small proportion of the establishments interviewed in the 1993 wave were also interviewed in 1999.
} 
Even though the DMMO and EMMO surveys were conducted throughout the 1990s, our merged database (REPONSE-DMMO-EMMO) provided by the French Ministry of Labor contains only information for 1998. We are thus forced to adopt a cross-sectional approach, in which we make extensive use of the rich data provided by REPONSE on establishments use of ICTs and HPWO practices. We analyze the correlation between the presence of these technological and organizational practices in an establishment and the labor flows of various occupational categories.

\subsection{Gross labor flows, entries, exits, net flows and churning flows}

The full DMMO-EMMO surveys include detailed information on labor flows by occupation. In order to measure the influence of ICTs and HPWOPs on job stability, we use various indicators:

Gross labor flows of occupation $i=\frac{\text { Hirings }+ \text { Firings of occupation } i \text { in the establishment }}{\text { Total number of occupation } i \text { in the establishment }}$

Entries of occupation $i=\frac{\text { Hirings of occupation } i \text { in the establishment }}{\text { Total number of occupation } i \text { in the establishment }}$, Exits of occupation $i=\frac{\text { Firings of occupation } i \text { in the establishment }}{\text { Total number of occupation } i \text { in the establishment }}$,

Net labor flows of occupation $i=\frac{\text { Hirings - Firings of occupation } i \text { in the establishment }}{\text { Total number of occupation } i \text { in the establishment }}$,

- Finally, the churning flows is normally considered as the most accurate measure of job instability, since it captures the difference between the gross labor flows (hirings+firings) and the net job creation/destruction (absolute value of the difference between the number of hirings and firings). This differential stands for the number of times that the establishments has replaced one worker by another one occupying exactly the same position.

$$
\text { Churning flows of occupation } i=\frac{\text { Hirings }+ \text { Firings }-\mid \text { Hirings }- \text { Firings } \mid \text { occupation } i}{\text { Total number of occupation } i} \text {, }
$$


where "occupation $i$ " corresponds to: managers $^{6}$ (MANAGERS), intermediate professionals 7 (INT. PROFES.), employees ${ }^{8}$ (EMPLOYEES), manual workers ${ }^{9}$ (WORKERS) and all workers (TOTAL). Remark that all the indicators are defined in relative terms so as to take into account the size of the establishment. On the other hand, it must be noticed that entries and exits from an establishment may be towards another establishment belonging to the same company.

\subsection{Technological variables}

New technologies, especially ICTs, are widely used in French establishments. We define three dummy variables capturing the "large-scale use" of computers (COMPUTER), network systems (NET) and internet (INTERNET) by establishments ${ }^{10}$.

In addition, automated production is represented by the dummy variable CHAIN, which captures the use of Taylorist production systems (robots, computer-assisted systems, etc.).

\subsection{Organizational variables}

To measure the effects of HPWO practices we consider six different variables. AUTONOMOUS and PROJECT equal unity when more than $20 \%$ of the workforce are members, respectively, of autonomous production teams or project groups. QUALITY and J.I.T. are dummies capturing whether the establishment practices, respectively, internal total quality control procedures or just-in-time production methods. ROTATION has a unitary value when the majority of workers rotates between tasks within the establishment. Finally, the variable HIERARCHY captures whether the establishment has reduced the number of hierarchical levels and uses organizational practices that seek to promote worker participation in decision-making within the establishment.

\subsection{Other variables}

In our original regressions we control for other variables that can affect labor flows. These variables are:

\footnotetext{
${ }^{6}$ Executive and managerial positions inside the establishment.

${ }^{7}$ Technicians and foremen.

${ }^{8}$ Clerks, office workers and business employees.

${ }^{9}$ Qualified and not qualified manual workers.

${ }^{10}$ When dealing with computers we consider use to be "large-scale" when more than $50 \%$ of the workers use them. In contrast, for network systems and internet, the reference percentage is more than $20 \%$ of workers.
} 
- Union: Dummy variable taking the value 1 if the workers have a union representative in the establishment. It seems quite intuitive that the presence of unions promotes job stability inside the establishment as far as the union cares about employment and not only about wages.

- The evolution of the economic activity of the establishment during the last three years is represented by means of the following variables: increasing, strongly increasing, decreasing and strongly decreasing. We also define a dummy variable (Variation) taking the value 1 if the economic activity of the establishment has known an unusual variation (positive or negative) in 1998.

- Tech. change and Org. change are dummy variables capturing, respectively, whether the establishment has suffered an important technological or organizational change over the last three years.

- We control for the size of the establishment through two dummy variables capturing whether the establishment has between 20 and 50 workers (Size 20-50) or more than 500 workers (Size +500) (establishments with 50 to 500 employees are taken as reference).

- P. Employees, P. Technicians and P. Managers are the proportion of employees, technicians and managers in the establishment (the proportion of manual workers is the reference). P. Women is the proportion of women in the establishment and P. Contract is the proportion of fixed duration contracts in the establishment.

- Hours: Dummy variable adopting the unitary value when the firm has already implemented the reduction in the number of working hours to 35 hours per week.

- We control for 16 economic sectors: agriculture and fishing; agricultural and food industry; consumption industry; automobile industry; equipment industry; intermediary goods industry; energy sector; building sector; trade sector; transport sector; financial activity sector; housing activities; services to firms; services to individuals; education, health and social action; and the public administration.

In the final regressions reported in the paper we only keep the control variables arising as significant for at least one of the considered professional categories. 


\subsection{Descriptive statistics}

Tables 11 and 12 in Appendix B summarize the means and standard deviations of all variables included in our analysis. The occupational category with the largest gross labor flows in relative terms is white-collar staff, followed by managers, intermediate professionals and manual workers. However, whereas for managers and manual workers average net flows turn out to be negative, for white-collar workers and intermediate professionals they are positive. Most of the intermediate professionals gross flows result from the turnover of people, leading to very high churning flows. With regard to technological variables, production line systems are very widespread in French establishments; in contrast the degree of Internet penetration is low. As far as organizational practices are concerned, the commonest ones are reductions in the number of hierarchical levels, just-in-time production systems and the implementation of quality control procedures.

Table 13 in Appendix B presents the correlation matrix between the technological and organizational variables. It can be seen that new technologies and new organizational practices are usually positively correlated (complementary relationship). However, the rotation of workers between tasks and just-in-time production systems are negatively related to COMPUTER and NET. A negative correlation between autonomous production teams and COMPUTER is also observed.

\section{Econometric strategy}

We proceed now to estimate the following econometric model:

$$
Y_{i e t}=\alpha_{1} I_{i e t}+\alpha_{2} O_{i e t}+\alpha_{3} X_{i e t}+v_{i e t},
$$

where the dependent variable will be the gross labor flows, entries, exits, net labor flows and churning flows of managers, intermediary professions, clerks, manual workers, all workers. The vector $I_{i e t}$ contains all variables measuring the presence of information and communication technologies in the establishment (COMPUTER, NET, INTERNET and CHAIN). The vector $O_{i e t}$ includes all variables describing the use of HPWOP by the establishment (AUTONOMOUS, PROJECT, ROTATION, QUALITY, HIERARCHY and J.I.T.). Finally $X_{i t}$ includes the control variables described in section 2.4 
Our econometric analysis follows the standard stages for studying the establishments' labor flows:

- We first estimate equation (1) for each of our indicators of job stability using OLS. As previously remarked, the regressions presented in the paper contain the control variables that were significant for at least one professional category.

- Second, the high degree of intercorrelation among the explicative variables (see table 13) may lead to a multicolinearity problem i.e. the estimated coefficients present high standard deviations. To solve this problem we can either eliminate some explicative variables, in which case we are likely to loose some valuable information, or we can simply define sets of highly correlated practices ("clusters") and re-estimate equation (1) introducing them as explicative variables. We adopt the second solution.

Due to missing observations, our estimations concern only half of the initial sample (in the case of manual workers the number of observations falls to 1080 since tertiary activities do not have this occupational category). On the other hand, there must be other technological and organizational practices affecting labor flows that we are unable to capture due to data limitations. This bias is reflected in the low values of the adjusted $R^{2}$.

\section{Results}

\subsection{First estimations}

This section provides the main results by broad occupations. Summary estimates are reported in table 2. Tables 5-6 in appendix A give the complete estimations for all workers and for managers. Because we work with cross-sectional data, we only provide correlations that suggest same impacts. They reveal that the potential effects of technological and organizational variables differ, and are even contradictory, depending on the occupational category under analysis.

Turnover among managers seems positively affected by organizational practices that seek to empower production workers and strengthen their commitment to the firm, such as autonomous production teams or project groups. These practices lead to increases in hirings and firings, 
positively influencing churning flows while reducing net flows. On the other hand, the implementation of total quality control procedures is negatively correlated with managers labor flows (both entries and exits) and decreases churning.

Reductions in the number of hierarchical levels are negatively related to the hiring rate (and thus gross flows) of intermediate professionals as well as to their churning flows. This result also holds for clerical workers whose hiring rate, firing rate, net flows and churning flows are negatively influenced by the variable HIERARCHY. In contrast, the large-scale use of computers in the establishment is positively related to the churning flows of clerical workers via the increase in hirings and firings.

Job stability of manual workers seems positively affected by the use of traditional production systems (CHAIN). These production methods are negatively correlated to the turnover of workers (churning) due to the reduction in hirings and firings. Simultaneously, the use of network systems has a positive impact on manual workers' gross labor flows as well as on churning flows (via the hiring rate).

The results for the total workforce in the establishment reflect the observed heterogeneity by occupation (table 6). Only 2 correlations are significant. The large-scale use of computers positively influences the hiring and firing rates, encouraging increased replacement of workers (churning). The rotation of workers among different tasks is also positively correlated to job instability via the exit rate, whereas the reduction in the number of hierarchical levels has a negative impact on hirings, firings and thus on turnover.

In sum, our estimates suggest that, since the use of ICTs requires certain skills, their presence is positively related to turnover among clerical and manual workers, who do not have the required skills. Moreover, a reduction in the use of production line systems positively influences job instability (churning) among manual workers. On the other hand, the fact that most HPWO practices aim to increase the motivation, participation and productivity of blue-collar workers (production workers) explains their positive effect on churning flows among managers and their negative effect on turnover among intermediate professionals and clerical workers (impact of HIERARCHY). 
Table 2: Technology and organization versus labor flows, entries, exits, net changes and churning flows by occupation. French establishments 1998.

\begin{tabular}{|c|c|c|c|c|c|}
\hline MANAGERS & LABOR FLOWS & ENTRIES & EXITS & NET FLOWS & CHURNING \\
\hline $\begin{array}{l}\text { COMPUTER } \\
\text { NET } \\
\text { CHAIN } \\
\text { AUTONOMOUS } \\
\text { PROJECT } \\
\text { ROTATION } \\
\text { QUALITY } \\
\text { HIERARCHY } \\
\text { JUST TIME }\end{array}$ & $\begin{array}{c}-1.25 \\
(1.71) \\
-0.25 \\
(1.54) \\
-0.55 \\
(0.92) \\
2.56 \\
(1.47)^{*} \\
3.06 \\
(1.46)^{* *} \\
-1.59 \\
(1.37) \\
-2.66 \\
(1.35)^{* *} \\
0.88 \\
(0.88) \\
1.12 \\
(0.73) \\
\end{array}$ & $\begin{array}{c}-0.61 \\
(0.86) \\
-0.13 \\
(0.77) \\
-0.27 \\
(0.46) \\
1.27 \\
(0.74)^{*} \\
1.53 \\
(0.73)^{* *} \\
-0.79 \\
(0.68) \\
-1.34 \\
(0.68)^{* *} \\
0.43 \\
(0.44) \\
0.57 \\
(0.37) \\
\end{array}$ & $\begin{array}{c}-0.61 \\
(0.86) \\
-0.13 \\
(0.77) \\
-0.28 \\
(0.46) \\
1.30 \\
(0.74)^{*} \\
1.52 \\
(0.73)^{* *} \\
-0.79 \\
(0.69) \\
-1.36 \\
(0.68)^{* *} \\
0.45 \\
(0.44) \\
0.57 \\
(0.37) \\
\end{array}$ & $\begin{array}{c}0.00 \\
(0.02) \\
0.00 \\
(0.02) \\
0.02 \\
(0.01)^{*} \\
-0.03 \\
(0.02)^{*} \\
0.01 \\
(0.02) \\
-0.01 \\
(0.02) \\
0.02 \\
(0.02) \\
-0.02 \\
(0.01)^{* *} \\
0.00 \\
(0.01) \\
\end{array}$ & $\begin{array}{c}-1.24 \\
(1.72) \\
-0.27 \\
(1.54) \\
-0.53 \\
(0.93)^{*} \\
2.56 \\
(1.48)^{* *} \\
3.06 \\
(1.47) \\
-1.60 \\
(1.37) \\
-2.66 \\
(1.35)^{* *} \\
0.87 \\
(0.88) \\
1.13 \\
(0.73) \\
\end{array}$ \\
\hline INTERMED.PROF. & LABOR FLOWS & ENTRIES & EXITS & NET FLOWS & CHURNING \\
\hline $\begin{array}{l}\text { COMPUTER } \\
\text { NET } \\
\text { CHAIN } \\
\text { AUTONOMOUS } \\
\text { PROJECT } \\
\text { ROTATION } \\
\text { QUALITY } \\
\text { HIERARCHY } \\
\text { JUST TIME }\end{array}$ & $\begin{array}{c}0.23 \\
(0.23) \\
-0.01 \\
(0.21) \\
0.06 \\
(0.12) \\
0.14 \\
(0.20) \\
-0.28 \\
(0.19) \\
0.18 \\
(0.18) \\
0.18 \\
(0.18) \\
-0.20 \\
(0.12)^{*} \\
-0.04 \\
(0.10) \\
\end{array}$ & $\begin{array}{c}0.14 \\
(0.12) \\
-0.02 \\
(0.11) \\
0.04 \\
(0.06) \\
0.05 \\
(0.10) \\
-0.14 \\
(0.10) \\
0.09 \\
(0.09) \\
0.06 \\
(0.09) \\
-0.11 \\
(0.06)^{*} \\
-0.02 \\
(0.05) \\
\end{array}$ & $\begin{array}{c}0.10 \\
(0.11) \\
0.00 \\
(0.10) \\
0.02 \\
(0.06) \\
0.08 \\
(0.10) \\
-0.14 \\
(0.10) \\
0.09 \\
(0.09) \\
0.12 \\
(0.09) \\
-0.09 \\
(0.06) \\
-0.02 \\
(0.05) \\
\end{array}$ & $\begin{array}{c}0.04 \\
(0.04) \\
-0.02 \\
(0.03) \\
0.02 \\
(0.02) \\
-0.03 \\
(0.03) \\
0.00 \\
(0.03) \\
0.00 \\
(0.03) \\
-0.06 \\
(0.03)^{* *} \\
-0.01 \\
(0.02) \\
0.00 \\
(0.02) \\
\end{array}$ & $\begin{array}{c}0.18 \\
(0.22) \\
0.03 \\
(0.20) \\
0.09 \\
(0.12) \\
0.14 \\
(0.19) \\
-0.27 \\
(0.19) \\
0.14 \\
(0.18) \\
0.18 \\
(0.17) \\
-0.20 \\
(0.11)^{*} \\
-0.04 \\
(0.09) \\
\end{array}$ \\
\hline CLERKS & LABOR FLOWS & ENTRIES & EXITS & NET FLOWS & CHURNING \\
\hline $\begin{array}{l}\text { COMPUTER } \\
\text { NET } \\
\text { CHAIN } \\
\text { AUTONOMOUS } \\
\text { PROJECT } \\
\text { ROTATION } \\
\text { QUALITY } \\
\text { HIERARCHY } \\
\text { JUST TIME }\end{array}$ & $\begin{array}{c}0.78 \\
(0.36)^{* *} \\
-0.06 \\
(0.33) \\
-0.20 \\
(0.20) \\
-0.17 \\
(0.32) \\
-0.20 \\
(0.31) \\
0.40 \\
(0.29) \\
0.00 \\
(0.29) \\
-0.41 \\
(0.19)^{* *} \\
-0.02 \\
(0.16) \\
\end{array}$ & $\begin{array}{c}0.41 \\
(0.20)^{* *} \\
-0.03 \\
(0.18) \\
-0.08 \\
(0.11) \\
-0.12 \\
(0.17) \\
-0.10 \\
(0.17) \\
0.18 \\
(0.16) \\
0.03 \\
(0.16) \\
-0.24 \\
(0.10)^{* *} \\
-0.03 \\
(0.09) \\
\end{array}$ & $\begin{array}{c}0.36 \\
(0.17)^{* *} \\
-0.03 \\
(0.15) \\
-0.12 \\
(0.09) \\
-0.05 \\
(0.15) \\
-0.10 \\
(0.15) \\
0.21 \\
(0.14) \\
-0.02 \\
(0.13) \\
-0.18 \\
(0.09)^{* *} \\
0.00 \\
(0.07) \\
\end{array}$ & $\begin{array}{c}0.05 \\
(0.07) \\
0.00 \\
(0.06) \\
0.04 \\
(0.04) \\
-0.07 \\
(0.06) \\
-0.01 \\
(0.06) \\
-0.03 \\
(0.06) \\
0.05 \\
(0.05) \\
-0.06 \\
(0.04)^{*} \\
-0.02 \\
(0.03) \\
\end{array}$ & $\begin{array}{c}0.74 \\
(0.34)^{* *} \\
-0.07 \\
(0.31) \\
-0.20 \\
(0.18) \\
-0.15 \\
(0.29) \\
-0.20 \\
(0.29) \\
0.41 \\
(0.27) \\
-0.04 \\
(0.27) \\
-0.36 \\
(0.17)^{* *} \\
-0.01 \\
(0.15) \\
\end{array}$ \\
\hline MANUAL WORK. & LABOR FLOWS & ENTRIES & EXITS & NET FLOWS & CHURNING \\
\hline $\begin{array}{l}\text { COMPUTER } \\
\text { NET } \\
\text { CHAIN } \\
\text { AUTONOMOUS } \\
\text { PROJECT } \\
\text { ROTATION } \\
\text { QUALITY } \\
\text { HIERARCHY } \\
\text { JUST TIME }\end{array}$ & $\begin{array}{c}0.08 \\
(0.21) \\
0.39 \\
(0.19)^{* *} \\
-0.26 \\
(0.11)^{* *} \\
-0.02 \\
(0.17) \\
-0.28 \\
(0.18) \\
-0.13 \\
(0.16) \\
-0.08 \\
(0.17) \\
0.05 \\
(0.11) \\
0.08 \\
(0.09) \\
\end{array}$ & $\begin{array}{c}0.02 \\
(0.10) \\
0.23 \\
(0.09)^{* * *} \\
-0.13 \\
(0.06)^{* *} \\
-0.01 \\
(0.08) \\
-0.15 \\
(0.09)^{*} \\
-0.06 \\
(0.08) \\
-0.08 \\
(0.08) \\
0.02 \\
(0.05) \\
0.04 \\
(0.04) \\
\end{array}$ & $\begin{array}{c}0.06 \\
(0.11) \\
0.16 \\
(0.10) \\
-0.14 \\
(0.06)^{* *} \\
-0.01 \\
(0.09) \\
-0.13 \\
(0.09) \\
-0.07 \\
(0.08) \\
0.00 \\
(0.09) \\
0.02 \\
(0.06) \\
0.04 \\
(0.05) \\
\end{array}$ & $\begin{array}{c}-0.03 \\
(0.05) \\
0.07 \\
(0.04)^{*} \\
0.01 \\
(0.03) \\
0.00 \\
(0.04) \\
-0.02 \\
(0.04) \\
0.00 \\
(0.04) \\
-0.07 \\
(0.04)^{* *} \\
0.00 \\
(0.02) \\
0.00 \\
(0.02) \\
\end{array}$ & $\begin{array}{c}0.04 \\
(0.18) \\
0.40 \\
(0.16)^{* * *} \\
-0.22 \\
(0.09)^{* *} \\
-0.02 \\
(0.14) \\
-0.24 \\
(0.15) \\
-0.11 \\
(0.13) \\
-0.09 \\
(0.14) \\
0.01 \\
(0.09) \\
0.06 \\
(0.07) \\
\end{array}$ \\
\hline
\end{tabular}

() Standard errors.

12

*Significant at $10 \%{ }^{* *}$ Significant at $5 \% . * * *$ Significant at $1 \%$

Source: REPONSE, DMMO and EMMO surveys. 


\subsection{Dealing with the multicolinearity problem}

The high degree of intercorrelation among the explicative variables (see table 13) indicates that empirical models estimating the impact of ICT and HPWO practices on gross labor flows, entries, exits, net flows and churning flows may yield unstable coefficients. To solve this problem a traditional approach used in the literature when only cross-sectional data are available involves defining sets of highly correlated practices (e.g., Ichniowski, Shaw, and Prennushi (1997)). In this paper, we consider two types of clusters differing in their economic interpretation.

- We analyze first the potential impact of what we will call "incremental organization" or "additive clusters". These sets of practices capture a kind of continuity (or intensity) in technological and organizational changes.

- Second, we consider clusters including complementary technological and organizational practices ("multiplicative clusters"). A consistent literature (e.g., Ichniowski, Shaw, and Prennushi (1997) or Askenazy and Gianella (2000)) claims that firms realize the largest productivity gains by adopting clusters of complementary practices. Thus it seems relevant to analyze the effect that these sets of interactive practices have on labor flows.

\subsubsection{The incremental organization}

The incremental organization cluster can be interpreted in economic terms as measuring the intensity of the use of technological and organizational practices. We define three sets of variables capturing practices that have similar objectives and are highly intercorrelated:

1. TECHNOLOGY: Cluster including the technological variables COMPUTER and NET. The presence of one of these practices is sufficient to guarantee the non nullity of TECHNOLOGY.

2. TEAMWORK: Set of organizational variables including all practices tending towards the delegation of responsibilities and the promotion of working teams. The non nullity of TEAMWORK is guaranteed by the presence of any of the following practices: AUTONOMOUS, PROJECT or HIERARCHY. 
3. FLEXIBILITY: Cluster covering all organizational practices stimulating a flexible job assignment (ROTATION and J.I.T.).

Table 3 summarizes the estimations obtained. Table 7 in Appendix A provides the detailed results for clerical workers and Table 8 those for all workers. We observe that workplace organizational practices favoring the delegation of responsibilities to lower hierarchical levels as well as the presence of work teams (TEAMWORK) are positively related to the increased hiring and firing of managers. Both tendencies have a negative impact on net flows, whereas churning flows are positively influenced. In contrast, quality control procedures continue to have a stabilizing effect on turnover among managers.

The set of organizational practices included in TEAMWORK is negatively correlated to gross and churning flows among intermediate professionals and clerical workers, via the reduction in the hiring and firing rates. It seems interesting to remark that the cluster capturing the intensity of the use of technological practices does not turn out to be significant for any of the stability indicators concerning clerical workers, while the individual variable COMPUTER had a positive influence on churning flows among white-collar workers (see Table 2).

Estimations for manual workers confirm our previous result. The variable TECHNOLOGY is positively correlated to hiring and firing rates (gross flows), reflecting the increased replacement of manual workers (churning). Conversely the use of traditional production line systems maintains its negative and significant coefficients for gross labor flows, entries, exits and churning flows.

Finally, at the establishment level, the use of ICTs is positively related to net flows, whereas the use of novel organizational practices favoring the delegation of responsibilities to lower hierarchical levels as well as the presence of work teams (TEAMWORK) has a negative impact on churning flows.

In sum, results in Table 3 mainly confirm the two general findings drawn from the analysis of Table 2. While the presence of ICTs is associated with higher turnover among manual workers, HPWO practices that seek to reduce hierarchical levels and encourage autonomous work groups are positively correlated to turnover among managers and negatively related to labor flows among intermediate professionals and clerical workers. 
Table 3: Additive clusters and labor flows, entries, exits, net flows and churning flows. French establishments 1998.

\begin{tabular}{|c|c|c|c|c|c|}
\hline MANAGERS & LABOR FLOWS & ENTRIES & EXITS & NET FLOWS & CHURNING \\
\hline $\begin{array}{l}\text { TECHNOLOGY } \\
\text { CHAIN } \\
\text { TEAMWORK } \\
\text { FLEXIBILITY } \\
\text { QUALITY }\end{array}$ & $\begin{array}{c}-0.66 \\
(0.96) \\
-0.47 \\
(0.92) \\
1.82 \\
(0.61)^{* * *} \\
0.46 \\
(0.62) \\
-2.50 \\
(1.35)^{*}\end{array}$ & $\begin{array}{c}-0.33 \\
(0.48) \\
-0.22 \\
(0.46) \\
0.90 \\
(0.31)^{* * *} \\
0.24 \\
(0.31) \\
-1.26 \\
(0.68)^{*}\end{array}$ & $\begin{array}{c}-0.33 \\
(0.48) \\
-0.24 \\
(0.46) \\
0.92 \\
(0.31)^{* * *} \\
0.24 \\
(0.31) \\
-1.28 \\
(0.68)^{*}\end{array}$ & $\begin{array}{c}0.00 \\
(0.01) \\
0.02 \\
(0.01)^{*} \\
-0.02 \\
(0.01)^{* *} \\
0.00 \\
(0.01) \\
0.02 \\
(0.02)\end{array}$ & $\begin{array}{c}-0.67 \\
(0.96) \\
-0.45 \\
(0.92) \\
1.81 \\
(0.61)^{* * *} \\
0.47 \\
(0.62) \\
-2.51 \\
(1.35)^{*}\end{array}$ \\
\hline $\begin{array}{l}\text { Obs. } \\
R^{2}\end{array}$ & $\begin{array}{l}1388 \\
0.04\end{array}$ & $\begin{array}{l}1384 \\
0.04\end{array}$ & $\begin{array}{l}1384 \\
0.04\end{array}$ & $\begin{array}{l}1384 \\
0.02\end{array}$ & $\begin{array}{l}1384 \\
0.04\end{array}$ \\
\hline INTERMED. PROF. & LABOR FLOWS & ENTRIES & EXITS & NET FLOWS & CHURNING \\
\hline $\begin{array}{l}\text { TECHNOLOGY } \\
\text { CHAIN } \\
\text { TEAMWORK } \\
\text { FLEXIBILITY } \\
\text { QUALITY }\end{array}$ & $\begin{array}{c}0.09 \\
(0.13) \\
0.05 \\
(0.12) \\
-0.15 \\
(0.08)^{*} \\
0.01 \\
(0.08) \\
0.17 \\
(0.18)\end{array}$ & $\begin{array}{c}0.05 \\
(0.07) \\
0.04 \\
(0.06) \\
-0.08 \\
(0.04)^{* *} \\
0.01 \\
(0.04) \\
0.05 \\
(0.09)\end{array}$ & $\begin{array}{c}0.04 \\
(0.06) \\
0.01 \\
(0.06) \\
-0.07 \\
(0.04)^{*} \\
0.01 \\
(0.04) \\
0.11 \\
(0.09)\end{array}$ & $\begin{array}{c}0.01 \\
(0.02) \\
0.02 \\
(0.02) \\
-0.01 \\
(0.01) \\
0.00 \\
(0.01) \\
-0.06 \\
(0.03)^{* *}\end{array}$ & $\begin{array}{c}0.09 \\
(0.12) \\
0.08 \\
(0.12) \\
-0.15 \\
(0.08)^{*} \\
0.01 \\
(0.08) \\
0.17 \\
(0.17)\end{array}$ \\
\hline $\begin{array}{l}\text { Obs. } \\
R^{2}\end{array}$ & $\begin{array}{l}1374 \\
0.15\end{array}$ & $\begin{array}{l}1371 \\
0.15\end{array}$ & $\begin{array}{l}1371 \\
0.14\end{array}$ & $\begin{array}{l}1371 \\
0.02\end{array}$ & $\begin{array}{l}1371 \\
0.14\end{array}$ \\
\hline CLERKS & LABOR FLOWS & ENTRIES & EXITS & NET FLOWS & CHURNING \\
\hline $\begin{array}{l}\text { TECHNOLOGY } \\
\text { CHAIN } \\
\text { TEAMWORK } \\
\text { FLEXIBILITY } \\
\text { QUALITY }\end{array}$ & $\begin{array}{c}0.33 \\
(0.20) \\
-0.22 \\
(0.20) \\
-0.32 \\
(0.13)^{* *} \\
0.07 \\
(0.13) \\
-0.03 \\
(0.29)\end{array}$ & $\begin{array}{c}0.18 \\
(0.11) \\
-0.09 \\
(0.11) \\
-0.19 \\
(0.07)^{* * *} \\
0.02 \\
(0.07) \\
0.02 \\
(0.16)\end{array}$ & $\begin{array}{c}0.15 \\
(0.10) \\
-0.13 \\
(0.09) \\
-0.13 \\
(0.06)^{* *} \\
0.05 \\
(0.06) \\
-0.03 \\
(0.13)\end{array}$ & $\begin{array}{c}0.03 \\
(0.04) \\
0.04 \\
(0.04) \\
-0.05 \\
(0.02)^{* *} \\
-0.03 \\
(0.03) \\
0.05 \\
(0.05)\end{array}$ & $\begin{array}{c}0.30 \\
(0.19) \\
-0.22 \\
(0.18) \\
-0.28 \\
(0.12)^{* *} \\
0.08 \\
(0.12) \\
-0.07 \\
(0.27)\end{array}$ \\
\hline $\begin{array}{l}\text { Obs. } \\
R^{2}\end{array}$ & $\begin{array}{l}1359 \\
0.07\end{array}$ & $\begin{array}{l}1355 \\
0.07\end{array}$ & $\begin{array}{l}1355 \\
0.08\end{array}$ & $\begin{array}{c}1355 \\
0.01\end{array}$ & $\begin{array}{l}1355 \\
0.07\end{array}$ \\
\hline MANUAL WORK. & LABOR FLOWS & ENTRIES & EXITS & NET FLOWS & CHURNING \\
\hline $\begin{array}{l}\text { TECHNOLOGY } \\
\text { CHAIN } \\
\text { TEAMWORK } \\
\text { FLEXIBILITY } \\
\text { QUALITY }\end{array}$ & $\begin{array}{c}0.24 \\
(0.12)^{* *} \\
-0.25 \\
(0.11)^{* *} \\
-0.04 \\
(0.07) \\
0.03 \\
(0.07) \\
-0.08 \\
(0.17)\end{array}$ & $\begin{array}{c}0.13 \\
(0.06)^{* *} \\
-0.12 \\
(0.05)^{* *} \\
-0.02 \\
(0.04) \\
0.02 \\
(0.04) \\
-0.07 \\
(0.08)\end{array}$ & $\begin{array}{c}0.11 \\
(0.06)^{*} \\
-0.13 \\
(0.06)^{* *} \\
-0.02 \\
(0.04) \\
0.01 \\
(0.04) \\
0.00 \\
(0.09)\end{array}$ & $\begin{array}{c}0.02 \\
(0.03) \\
0.01 \\
(0.03) \\
0.00 \\
(0.02) \\
0.00 \\
(0.02) \\
-0.07 \\
(0.04)^{*}\end{array}$ & $\begin{array}{c}0.23 \\
(0.10)^{* *} \\
-0.21 \\
(0.09)^{* *} \\
-0.05 \\
(0.06) \\
0.02 \\
(0.06) \\
-0.08 \\
(0.14)\end{array}$ \\
\hline $\begin{array}{l}\text { Obs. } \\
R^{2}\end{array}$ & $\begin{array}{c}1079 \\
0.09\end{array}$ & $\begin{array}{l}1076 \\
0.08\end{array}$ & $\begin{array}{c}1076 \\
0.09\end{array}$ & $\begin{array}{l}1076 \\
0.06\end{array}$ & $\begin{array}{c}1076 \\
0.09\end{array}$ \\
\hline
\end{tabular}

() Standard errors.

${ }^{*}$ Significant at $10 \%{ }^{* *}$ Significant at $5 \%{ }^{* * *}$ Significant at $1 \%$.

Source: REPONSE, DMMO and EMMO surveys. 


\subsubsection{The complementary relationships}

We consider here two sets of a priori complementary practices:

1. TEAMWORK*: Set of organizational variables including all practices tending towards the delegation of responsibilities and the promotion of working teams. The non nullity of TEAMWORK* is only guaranteed when the HPWO practices AUTONOMOUS, PROJECT and HIERARCHY are simultaneously present in the establishment.

2. ICT FLEXIBILITY: This cluster combines technological and organizational variables. It tries to capture the fact that the massive use of new technologies (COMPUTER) combined with flexible job assignment practices (ROTATION), normally acts in the same sense over labor flows.

Table 4 shows that the individual variables AUTONOMOUS, PROJECT and HIERARCHY lose their significance when considering managers, while the coefficient of the variable capturing their interactions (TEAMWORK*) becomes significant and positive. Thus the three HPWO practices reinforce each other, and their interaction is positively related to hiring and firing rates. Both impacts seem to compensate each other, since net flows could be not affected by TEAMWORK*, while churning flows are positively related to it. Quality control procedures continue to be negatively correlated to managers' turnover (reduced entries, exits and churning). As far as clerical workers are concerned, the combination of new technologies and flexible organizational practices (ICT FLEXIBILITY) is positively correlated to their gross labor flows, entries, exits and churning flows. The HPWO practice of delegating responsibilities to lower hierarchical levels (HIERARCHY) acts in the opposite direction, potentially reducing job instability. Finally, it should be noted that complementarities between technological and organizational variables are not significant for intermediate professionals and manual workers.

For the whole set of workers (Table 10), churning flows are positively correlated to the simultaneous presence of ICTs and flexible job assignment practices, via the increased hiring and firing rates. In contrast, ICT FLEXIBILITY does not have a significant effect on net flows, resulting only in higher turnover. Consequently, complementarities between technological and organizational practices must also be considered when analyzing the effect of ICTs and HPWO 
Table 4: Multiplicative clusters and labor flows, entries, exits, net flows and churning flows. French establishments 1998.

\begin{tabular}{|c|c|c|c|c|c|}
\hline MANAGERS & LABOR FLOWS & ENTRIES & EXITS & NET FLOWS & CHURNING \\
\hline COMPUTER & $\begin{array}{l}-1.08 \\
(1.87)\end{array}$ & $\begin{array}{l}-0.52 \\
(0.94)\end{array}$ & $\begin{array}{l}-0.52 \\
(0.94)\end{array}$ & $\begin{array}{c}0.00 \\
(0.02)\end{array}$ & $\begin{array}{l}-1.07 \\
(1.87)\end{array}$ \\
\hline NET & -0.44 & -0.23 & -0.23 & 0.00 & -0.46 \\
\hline CHAIN & $\begin{array}{l}(1.03) \\
-0.59\end{array}$ & $\begin{array}{l}(0.77) \\
-0.29\end{array}$ & $\begin{array}{l}(0.77) \\
-0.31\end{array}$ & $\begin{array}{l}(0.02) \\
0.02\end{array}$ & $\begin{array}{l}(1.53) \\
-0.57\end{array}$ \\
\hline AUTONOMOUS & $\begin{array}{l}(0.92) \\
-0.11\end{array}$ & $\begin{array}{l}(0.46) \\
-0.07\end{array}$ & $\begin{array}{l}(0.46) \\
-0.03\end{array}$ & $\begin{array}{l}(0.01)^{*} \\
-0.04\end{array}$ & $\begin{array}{l}(0.92) \\
-0.12\end{array}$ \\
\hline PROJECT & $\begin{array}{c}(1.67) \\
0.70\end{array}$ & $(0.84)$ & $(0.84)$ & $(0.02)^{* *}$ & $(1.68)$ \\
\hline PRUJECI & $(1.62)$ & $\begin{array}{l}0.35 \\
(0.81)\end{array}$ & $\begin{array}{l}0.34 \\
(0.81)\end{array}$ & $(0.02)$ & $\begin{array}{l}0.69 \\
(1.62)\end{array}$ \\
\hline ROTATION & $\begin{array}{l}-1.40 \\
(1.53)\end{array}$ & $\begin{array}{l}-0.69 \\
(0.76)\end{array}$ & $\begin{array}{l}-0.69 \\
(0.77)\end{array}$ & $\begin{array}{l}0.00 \\
(0.02)\end{array}$ & $\begin{array}{l}-1.41 \\
(1.53)\end{array}$ \\
\hline QUALITY & -2.47 & -1.24 & -1.26 & $\begin{array}{l}0.02 \\
(0.02)\end{array}$ & -2.47 \\
\hline HIERARCHY & 0.43 & 0.20 & 0.23 & $\begin{array}{l}(0.02) \\
-0.02 * *\end{array}$ & $\begin{array}{c}0.41 \\
0.88\end{array}$ \\
\hline JUST TIME & $\begin{array}{l}0.00 \\
0.97\end{array}$ & 0.50 & $\begin{array}{l}(0.44) \\
0.49\end{array}$ & $\begin{array}{l}(0.01) \times- \\
0.00\end{array}$ & $\begin{array}{l}(0.88) \\
0.99\end{array}$ \\
\hline TEAMWORK* & $\begin{array}{l}(0.73) \\
59.47\end{array}$ & $\begin{array}{c}(0.37) \\
2.98\end{array}$ & $\begin{array}{c}(0.37) \\
2.96\end{array}$ & $\begin{array}{c}(0.01) \\
0.01\end{array}$ & $\begin{array}{c}(0.73) \\
5.96\end{array}$ \\
\hline ICT FLEXIBILITY & $\begin{array}{c}(1.79)^{* * *} \\
-0.36 \\
(3.16)\end{array}$ & $\begin{array}{c}(0 . \overline{8} 9)^{* * *} \\
-0.20 \\
(1.58)\end{array}$ & $\begin{array}{c}(0 . \overline{90}) * * * \\
-0.20 \\
(1.59)\end{array}$ & $\begin{array}{l}(0.02) \\
0.00 \\
(0.04)\end{array}$ & $\begin{array}{c}(1.79)^{* * *} \\
-0.35 \\
(3.17)\end{array}$ \\
\hline INTERMED. PROF. & LABOR FLOWS & ENTRIES & EXITS & NET FLOWS & CHURNING \\
\hline COMPUTER & $\begin{array}{l}0.18 \\
(0.25)\end{array}$ & $\begin{array}{c}0.12 \\
(0.13)\end{array}$ & $\begin{array}{c}0.07 \\
(0.12)\end{array}$ & $\begin{array}{c}0.05 \\
(0.04)\end{array}$ & $\begin{array}{l}0.15 \\
(0.24)\end{array}$ \\
\hline NET & $\begin{array}{l}-0.01 \\
(0.21)\end{array}$ & $\begin{array}{l}-0.02 \\
(0.11)\end{array}$ & $\begin{array}{l}0.00 \\
0.10)\end{array}$ & $\begin{array}{l}-0.02 \\
(0.03)\end{array}$ & $\begin{array}{c}0.03 \\
0.20)\end{array}$ \\
\hline CHAIN & $\begin{array}{l}0.06 \\
(0.12)\end{array}$ & $\begin{array}{l}0.04 \\
(0.06)\end{array}$ & $\begin{array}{l}0.02 \\
0.06)\end{array}$ & $\begin{array}{l}0.02 \\
(0.02)\end{array}$ & $\begin{array}{l}0.09 \\
0.12)\end{array}$ \\
\hline AUTONOMOUS & $\begin{array}{l}0.11 \\
(0.22)\end{array}$ & $\begin{array}{l}0.03 \\
0.12)\end{array}$ & $\begin{array}{l}0.07 \\
0.11)\end{array}$ & $\begin{array}{l}-0.04 \\
(0.04)\end{array}$ & $\begin{array}{l}0.09 \\
0.21)\end{array}$ \\
\hline PROJECT & $\begin{array}{l}-0.31 \\
(0.22)\end{array}$ & $\begin{array}{l}-0.16 \\
(0.11)\end{array}$ & $\begin{array}{l}-0.15 \\
0.11)\end{array}$ & $\begin{array}{l}-0.01 \\
(0.04)\end{array}$ & $\begin{array}{l}-0.32 \\
(0.21)\end{array}$ \\
\hline ROTATION & $\begin{array}{l}0.14 \\
0.20)\end{array}$ & $\begin{array}{l}0.07 \\
(0.11)\end{array}$ & $\begin{array}{l}0.06 \\
0.10)\end{array}$ & $\begin{array}{l}0.01 \\
0.03)\end{array}$ & $\begin{array}{l}0.12 \\
0.20)\end{array}$ \\
\hline QUALITY & 0.18 & 0.06 & 0.12 & -0.06 & 0.18 \\
\hline HIERARCHY & $(-0.21$ * & $(-0.11$ * & $\begin{array}{l}-0.10 \\
(0.06)\end{array}$ & $\begin{array}{l}-0.01 \\
-0.02)\end{array}$ & $-0.21 *$ \\
\hline JUST TIME & $\begin{array}{l}-0.04 \\
(0.10)\end{array}$ & $\begin{array}{l}-0.02 \\
(0.05)\end{array}$ & $\begin{array}{l}-0.02 \\
(0.05)\end{array}$ & $\begin{array}{l}0.00 \\
(0.02)\end{array}$ & $\begin{array}{l}-0.04 \\
(0.09)\end{array}$ \\
\hline TEAMWORK* & $\begin{array}{l}0.07 \\
(0.24)\end{array}$ & 0.04 & 0.03 & $\begin{array}{l}0.02 \\
(0.04)\end{array}$ & 0.11 \\
\hline ICT FLEXIBILITY & $\begin{array}{c}0.23) \\
(0.42)\end{array}$ & $\begin{array}{c}0.10 \\
(0.22)\end{array}$ & $\begin{array}{l}(0.12) \\
0.121)\end{array}$ & $\begin{array}{l}(0.04) \\
-0.02 \\
(0.07)\end{array}$ & $\begin{array}{l}0.10) \\
0.41)\end{array}$ \\
\hline CLERKS & LABOR FLOWS & ENTRIES & EXITS & NET FLOWS & CHURNING \\
\hline COMPUTER & $\begin{array}{l}0.25 \\
(0.40)\end{array}$ & $\begin{array}{l}0.12 \\
(0.22)\end{array}$ & $\begin{array}{l}0.11 \\
0.19)\end{array}$ & $\begin{array}{l}0.01 \\
0.08)\end{array}$ & $\begin{array}{l}0.23 \\
0.37)\end{array}$ \\
\hline NET & $\begin{array}{l}0.06 \\
-0.06\end{array}$ & $\begin{array}{l}-0.03 \\
-0.03\end{array}$ & -0.03 & 0.00 & -0.06 \\
\hline CHAIN & $\begin{array}{l}-0.20 \\
-0.20\end{array}$ & $\begin{array}{l}(0.18) \\
-0.08\end{array}$ & $\begin{array}{l}(0.15) \\
-0.12\end{array}$ & $\begin{array}{c}(0.06) \\
0.04\end{array}$ & $\begin{array}{l}(0.31) \\
-0.20\end{array}$ \\
\hline AUTONOMOUS & $\begin{array}{l}(0.20) \\
-0.34\end{array}$ & $\begin{array}{l}(0.11) \\
-0.22\end{array}$ & $\begin{array}{l}(0.09) \\
-0.13\end{array}$ & $\begin{array}{l}(0.04) \\
-0.09\end{array}$ & $\begin{array}{l}(0.18) \\
-0.29\end{array}$ \\
\hline PBOJECT & $(0.36)$ & $(0.20)$ & $(0.17)$ & $(0.07)$ & $(0.33)$ \\
\hline PROJECT & $\begin{array}{l}-0.37 \\
(0.35)\end{array}$ & $\begin{array}{l}-0.20 \\
(0.19)\end{array}$ & $\begin{array}{l}-0.17 \\
(0.16)\end{array}$ & $\begin{array}{l}-0.03 \\
(0.07)\end{array}$ & $\begin{array}{l}-0.34 \\
(0.32)\end{array}$ \\
\hline ROTATION & $\begin{array}{l}-0.08 \\
(0.32)\end{array}$ & $\begin{array}{l}-0.08 \\
(0.18)\end{array}$ & $\begin{array}{l}-0.01 \\
(0.15)\end{array}$ & $\begin{array}{l}-0.07 \\
(0.06)\end{array}$ & $\begin{array}{l}-0.05 \\
(0.30)\end{array}$ \\
\hline QUALITY & $\begin{array}{l}0.00 \\
0.20)\end{array}$ & 0.03 & $\begin{array}{l}-0.02 \\
-0.13)\end{array}$ & 0.05 & $\begin{array}{l}-0.04 \\
-0.27)\end{array}$ \\
\hline HIERARCHY & $\begin{array}{l}(0.29) \\
-0.45\end{array}$ & -0.26 & $\begin{array}{l}(0.13) \\
-0.19\end{array}$ & $\begin{array}{l}(0.05) \\
-0.07\end{array}$ & $\begin{array}{l}(0.27) \\
-0.39\end{array}$ \\
\hline JUST TIME & $\begin{array}{l}(0.19)^{*} \\
-0.05\end{array}$ & $\begin{array}{l}(0.10)^{* * *} \\
-0.04\end{array}$ & $\begin{array}{l}(0.09)^{* *} \\
-0.01\end{array}$ & $\begin{array}{l}(0.04)^{*} \\
-0.03\end{array}$ & $\begin{array}{l}(0.18)^{* *} \\
-0.03\end{array}$ \\
\hline TEAMWORK* & $\begin{array}{c}(0.16) \\
0.38\end{array}$ & $\begin{array}{c}(0.09) \\
0.22\end{array}$ & $\begin{array}{l}(0.07) \\
0.17\end{array}$ & $\begin{array}{c}(0.03) \\
0.05\end{array}$ & $\begin{array}{c}(0.15) \\
0.34\end{array}$ \\
\hline ICT FLEXIBILITY & $\begin{array}{c}(0.38) \\
2.28 \\
(0.68)^{* * *}\end{array}$ & $\begin{array}{c}(0.21) \\
1.23 \\
(0.27) * * *\end{array}$ & $\begin{array}{c}(0.18) \\
1.06 \\
(0.32) * * *\end{array}$ & $\begin{array}{l}(0.07) \\
0.17 \\
0.13)\end{array}$ & $\begin{array}{l}(0.36) \\
2.16 \\
2.16 * *\end{array}$ \\
\hline MANUAL WORK. & LABOR FLOWS & ENTRIES & EXITS & NET FLOWS & CHURNING \\
\hline COMPUTER & $\begin{array}{l}0.17 \\
(0.24)\end{array}$ & $\begin{array}{l}0.05 \\
(0.12)\end{array}$ & $\begin{array}{c}0.12 \\
0.13)\end{array}$ & $\begin{array}{l}-0.07 \\
(0.05)\end{array}$ & $\begin{array}{l}0.08 \\
(0.20)\end{array}$ \\
\hline NET & $0.39 * *$ & $0.23 * *$ & 0.16 & 0.07 & 0.40 \\
\hline CHAIN & -0.26 & $\begin{array}{ll}(0.09) \\
-0.13\end{array}$ & $\begin{array}{l}(0.10) \\
-0.14\end{array}$ & $\begin{array}{l}(0.04)^{*} \\
0.01\end{array}$ & $\begin{array}{l}(0.16) \\
-0.22\end{array}$ \\
\hline AUTONOMOUS & $\begin{array}{l}(0.11)^{* *} \\
-0.05\end{array}$ & $\begin{array}{c}(0.06)^{* *} \\
-0.03\end{array}$ & $\begin{array}{l}(0.06)^{* *} \\
-0.02\end{array}$ & $\begin{array}{l}(0.03) \\
-0.02)\end{array}$ & $\begin{array}{l}(0.09)^{* *} \\
-0.06\end{array}$ \\
\hline PROIECT & $(0.19)$ & $(0.10)$ & $(0.10)$ & $(0.04)$ & $(0.16)$ \\
\hline PROJECT & $\begin{array}{l}-0.31 \\
(0.20)\end{array}$ & $(0.10)^{-0} *$ & $\begin{array}{l}-0.14 \\
(0.11)\end{array}$ & $\begin{array}{l}-0.03 \\
(0.05)\end{array}$ & $(0.17)^{-0}$ \\
\hline ROTATION & $\begin{array}{l}-0.07 \\
(0.17)\end{array}$ & $\begin{array}{l}-0.04 \\
(0.09)\end{array}$ & $\begin{array}{l}-0.03 \\
(0.09)\end{array}$ & $\begin{array}{l}-0.02 \\
(0.04)\end{array}$ & $\begin{array}{l}-0.08 \\
0.15)\end{array}$ \\
\hline QUALITY & $\begin{array}{l}-0.08 \\
(0.17)\end{array}$ & $\begin{array}{l}-0.07 \\
(0.08)\end{array}$ & $\begin{array}{l}0.00 \\
(0.09)\end{array}$ & -0.07 & -0.08 \\
\hline HIERARCHY & $\begin{array}{l}(0.17) \\
0.05\end{array}$ & $\begin{array}{c}(0.08) \\
0.02\end{array}$ & $\begin{array}{c}(0.09) \\
0.02\end{array}$ & $\begin{array}{c}(0.04)^{* *} \\
0.00\end{array}$ & $\begin{array}{c}(0.14) \\
0.01\end{array}$ \\
\hline JUST TIME & $\begin{array}{c}(0.11) \\
0.08\end{array}$ & $\begin{array}{c}(0.05) \\
0.04\end{array}$ & $\begin{array}{c}(0.06) \\
0.04\end{array}$ & $\begin{array}{c}(0.02) \\
0.00\end{array}$ & $\begin{array}{c}(0.09) \\
0.06\end{array}$ \\
\hline TF A MWORL & $\begin{array}{c}(0.09) \\
0.08\end{array}$ & $(0.04)$ & $(0.05)$ & $(0.02)$ & $(0.07)$ \\
\hline TEAMWORK & $\begin{array}{l}0.08 \\
(0.21)\end{array}$ & $\begin{array}{l}0107 \\
0.11)\end{array}$ & $\begin{array}{l}0.02 \\
(0.11)\end{array}$ & $\begin{array}{l}0.03 \\
(0.05)\end{array}$ & $\begin{array}{l}0.09 \\
(0.18)\end{array}$ \\
\hline ICT FLEXIBILITY & $\begin{array}{l}-0.31 \\
(0.40)\end{array}$ & $\begin{array}{l}-0.09 \\
(0.20)\end{array}$ & $\begin{array}{l}-0.22 \\
(0.21)\end{array}$ & $\begin{array}{l}0.12 \\
(0.09)\end{array}$ & $\begin{array}{l}-0.15 \\
(0.34)\end{array}$ \\
\hline
\end{tabular}


practices on job stability. Overall, however, these estimations are consistent with the results of the 2 previous sections.

\section{Conclusion}

The main objective of this paper has been to gain insights in one aspect that is little investigated in the existing literature, namely the effect of ICTs and HPWO practices on job stability. Working with a French database covering more than 2,500 establishments we have analyzed the relationship between technological and organizational practices inside firms and various indicators of job stability (gross labor flows, hiring rate, firing rate, net labor flows and churning flows) observed for different occupational categories.

Our findings reveal that the presence of ICTs is positively correlated to aggregate job turnover as well as to that of manual workers, while the effects on clerical workers' job stability are less clear. With regard to the impact of HPWO practices, we conclude that when a new organizational practice transfers increased "power" to a particular occupational category (for example delayering transfers more responsibilities to lower hierarchical levels while the implementation of quality control procedures requires skilled staff) it is also negatively correlated to turnover in this category. Most of the suggested impact concerns churning flows, while net employment seems quantitatively less affected.

All in all, technological and organizational changes result in increased heterogeneity of labor flows among occupational categories, but without any of them individually having a determining effect on aggregate turnover (apart from the use of computers, which seems positively related to churning flows). Only the simultaneous presence of flexible organizational practices and computers displays a systematic positive coefficient for gross labor flows, entries, exits and churning flows. In sum, the heterogeneity of effects among the various occupational categories is likely to be the main factor responsible for the increasing feeling of job instability experienced by workers in recent years. 


\section{References}

Abowd, J., and F. Kramarz. 2003. "The Costs of Hiring and Separations." Labour Economics $10(5): 499-530$.

Askenazy, P., and C. Gianella. 2000. "Le Paradoxe de Productivité: les Changements Organisationnels, facteur complémentaire à l'informatisation." Economie et Statistique 9/10 (339-340): 219-242.

Bauer, T., and S. Bender. 2004. "Technological Change, Organizational Change, and Job Turnover." Labour Economics 11, no. 265-292.

Bresnahan, T.F., E. Brynjolfsson, and L.M. Hitt. 2002. "Information Technology, Workplace organization, and the Demand for skilled Labor: Firm-Level Evidence." The Quarterly Journal of Economics 117 (1): 339-376.

Burgess, S.M., J. Lane, and D. Stevens. 2000. "Job Flows , Worker Flows, and Churning." Journal of Labor Economics 18 (3): 473-502 (July).

Burgess, S.M., and S. Nickell. 1990. "Labour Turnover in UK Manufacturing." Economica 57 (227): 295-317 (August).

Givord, P., and E. Maurin. 2004. "Changes in job security and their causes: An empirical analysis for France, 1982-2002.” European Economic Review 48 (3): 595-615 (June).

Greenan, N., and D. Guellec. 2000. "Technological Innovation and Employment Reallocation." Labour 14 (4): 547-565.

Hamermesh, D., W.H.J. Hassink, and J.C. Van-Ours. 1996. "Job Turnover and Labor Turnover: A Taxonomy of Employment Dynamics." Annales d'Économie et de Statistique 41/42:2139.

Ichniowski, C., K. Shaw, and G. Prennushi. 1997. "The Effects of Human Resource Management Practices on Productivity: A Study of Steel Finishing Lines." The American Economic Review 87 (3): 291-313 (June).

Ilmakunnas, P., and M. Maliranta. 2003. "Worker Inflow, Outflow and Churning." William Davidson Institute Working Paper, no. 611. 
Krusell, P., L.E. Ohanian, J.V. Rios-Rull, and G.L. Violante. 2000. "Capital skill complementarity and inequality: A macroeconomic analysis." Econometrica 68 (5): 1029-53.

Machin, S., and J. Van Reenen. 1998. "Technology and changes in skill structure: Evidence from seven OECD countries." Quarterly Journal of Economics 113:1215-44.

Michelacci, C., and D. Lopez-Salido. 2004. "Technology Shocks and Job Flows." CEPR Discussion Paper, no. 4426.

Neumark, D., D. Polsky, and D. Hansen. 1999. "Has Job Stability Declined Yet? New Evidence for the 1990s." Journal of Labor Economics 4, no. S29-S64 (October).

Neumark, D., and D. Reed. 2004. "Employment Relationships in the New Economy." Labour Economics 11 (1): 1-31 (February). 
6 Appendix A: Econometric results

OLS estimations 
Table 5: Determinants of managers' labor flows, entries, exits, net flows and churning flows. French establishments 1998.

\begin{tabular}{|c|c|c|c|c|c|}
\hline MANAGERS & LABOR FLOWS & ENTRIES & EXITS & NET FLOWS & CHURNING \\
\hline \multirow[t]{2}{*}{ COMPUTER } & -1.25 & -0.61 & -0.61 & 0.00 & -1.24 \\
\hline & $(1.71)$ & $(0.86)$ & $(0.86)$ & $(0.02)$ & $(1.72)$ \\
\hline \multirow[t]{2}{*}{ NET } & -0.25 & -0.13 & -0.13 & 0.00 & -0.27 \\
\hline & $(1.54)$ & $(0.77)$ & $(0.77)$ & $(0.02)$ & $(1.54)$ \\
\hline \multirow[t]{2}{*}{ CHAIN } & -0.55 & -0.27 & -0.28 & 0.02 & -0.53 \\
\hline & $(0.92)$ & $(0.46)$ & $(0.46)$ & $(0.01)^{*}$ & $(0.93) *$ \\
\hline \multirow[t]{2}{*}{ AUTONOMOUS } & 2.56 & 1.27 & 1.30 & -0.03 & 2.56 \\
\hline & $(1.47)^{*}$ & $(0.74)^{*}$ & $(0.74)^{*}$ & $(0.02)^{*}$ & $(1.48)^{* *}$ \\
\hline \multirow[t]{2}{*}{ PROJECT } & 3.06 & 1.53 & 1.52 & 0.01 & 3.06 \\
\hline & $(1.46)^{* *}$ & $(0.73)^{* *}$ & $(0.73)^{* *}$ & $(0.02)$ & $(1.47)$ \\
\hline \multirow[t]{2}{*}{ ROTATION } & -1.59 & -0.79 & -0.79 & -0.01 & -1.60 \\
\hline & $(1.37)$ & $(0.68)$ & $(0.69)$ & $(0.02)$ & $(1.37)$ \\
\hline \multirow[t]{2}{*}{ QUALITY } & -2.66 & -1.34 & -1.36 & 0.02 & -2.66 \\
\hline & $(1.35)^{* *}$ & $(0.68)^{* *}$ & $(0.68)^{* *}$ & $(0.02)$ & $(1.35)^{* *}$ \\
\hline \multirow[t]{2}{*}{ HIERARCHY } & 0.88 & 0.43 & 0.45 & -0.02 & 0.87 \\
\hline & $(0.88)$ & $(0.44)$ & $(0.44)$ & $(0.01)^{* *}$ & $(0.88)$ \\
\hline \multirow[t]{2}{*}{ JUST TIME } & 1.12 & 0.57 & 0.57 & 0.00 & 1.13 \\
\hline & $(0.73)$ & $(0.37)$ & $(0.37)$ & $(0.01)$ & $(0.73)$ \\
\hline \multirow[t]{2}{*}{ Variation } & 0.95 & 0.48 & 0.49 & -0.01 & 0.96 \\
\hline & $(1.22)$ & $(0.61)$ & $(0.61)$ & $(0.01)$ & $(1.22)$ \\
\hline \multirow[t]{2}{*}{ Tech. change } & -1.35 & -0.65 & -0.66 & 0.02 & -1.31 \\
\hline & $(1.53)$ & $(0.77)$ & $(0.77)$ & $(0.02)$ & $(1.53)$ \\
\hline \multirow[t]{2}{*}{ Org. change } & 1.17 & 0.57 & 0.59 & -0.01 & 1.14 \\
\hline & $(1.26)$ & $(0.63)$ & $(0.63)$ & $(0.01)$ & $(1.26)$ \\
\hline \multirow[t]{2}{*}{ Increasing } & -0.45 & -0.22 & -0.24 & 0.03 & -0.45 \\
\hline & $(1.77)$ & $(0.89)$ & $(0.89)$ & $(0.02)$ & $(1.77)$ \\
\hline \multirow[t]{2}{*}{ Decreasing } & 5.00 & 2.51 & 2.50 & 0.01 & 4.99 \\
\hline & $(1.85)^{* * *}$ & $(0.93)^{* * *}$ & $(0.93)^{* * *}$ & $(0.02)$ & $(1.85)^{* * *}$ \\
\hline \multirow[t]{2}{*}{ Hours } & 4.21 & 2.14 & 2.12 & 0.02 & 4.29 \\
\hline & $(1.76)^{* *}$ & $(0.88)^{* *}$ & $(0.88)^{* *}$ & $(0.02)$ & $(1.77)^{* *}$ \\
\hline \multirow[t]{2}{*}{ P. Employees } & 4.95 & 2.47 & 2.54 & -0.07 & 4.94 \\
\hline & $(2.96)^{*}$ & $(1.48)^{*}$ & $(1.49)^{*}$ & $(0.03)^{* *}$ & $(2.97)^{*}$ \\
\hline \multirow[t]{2}{*}{ P. Technicians } & -0.34 & -0.16 & -0.14 & -0.02 & -0.29 \\
\hline & $(4.48)$ & $(2.24)$ & $(2.25)$ & $(0.05)$ & $(4.49)$ \\
\hline \multirow[t]{2}{*}{ P. Managers } & -3.73 & -1.79 & -1.93 & 0.14 & -3.55 \\
\hline & $(5.26)$ & $(2.63)$ & $(2.64)$ & $(0.06)^{* *}$ & $(5.26)$ \\
\hline \multirow[t]{2}{*}{ P. Women } & 0.40 & 0.19 & 0.13 & 0.05 & 0.31 \\
\hline & $(2.73)$ & $(1.37)$ & $(1.38)$ & $(0.03)^{*}$ & $(2.75)$ \\
\hline \multirow[t]{2}{*}{ P. Contract } & -6.67 & -3.27 & -3.41 & 0.13 & $\begin{array}{l}-6.59 \\
(5.59)\end{array}$ \\
\hline & $\begin{array}{l}(5.58) \\
-1.62\end{array}$ & $(2.79)$ & $(2.80)$ & $(0.06)^{* *}$ & $\begin{array}{l}(5.59) \\
-1.77\end{array}$ \\
\hline Constant & $\begin{array}{l}-1.62 \\
(2.47)\end{array}$ & $\begin{array}{l}-0.86 \\
(1.24)\end{array}$ & $\begin{array}{l}-0.79 \\
(1.24)\end{array}$ & $\begin{array}{c}-0.06 \\
(0.03)^{* *}\end{array}$ & $\begin{array}{l}-1.77 \\
(2.47)\end{array}$ \\
\hline Sectors (10) & YES & YES & YES & YES & YES \\
\hline Obs. & 1388 & 1384 & 1384 & 1384 & 1384 \\
\hline$R^{2}$ & 0.04 & 0.04 & 0.04 & 0.02 & 0.04 \\
\hline
\end{tabular}

() Standard errors.

For Sectors we write in parentheses the number of economic sectors included in the regression.

${ }^{*}$ Significant at $10 \%$.**Significant at $5 \%{ }^{* * *}$ Significant at $1 \%$.

Source: REPONSE, DMMO and EMMO surveys. 
Table 6: Determinants of total labor flows, entries, exits, net flows and churning flows. French establishments 1998.

\begin{tabular}{|c|c|c|c|c|c|}
\hline TOTAL & LABOR FLOWS & ENTRIES & EXITS & NET FLOWS & CHURNING \\
\hline COMPUTER & $\begin{array}{c}0.23 \\
(0.11)^{* *}\end{array}$ & $\begin{array}{c}0.14 \\
(0.06)^{* *}\end{array}$ & $\begin{array}{c}0.14 \\
(0.06)^{* *}\end{array}$ & $\begin{array}{c}0.01 \\
(0.02)\end{array}$ & $\begin{array}{c}0.24 \\
(0.12)^{* *}\end{array}$ \\
\hline NET & $\begin{array}{c}-0.10 \\
(0.10)\end{array}$ & $\begin{array}{l}-0.05 \\
(0.06)\end{array}$ & $\begin{array}{l}-0.07 \\
(0.05)\end{array}$ & $\begin{array}{c}0.03 \\
(0.02)\end{array}$ & $\begin{array}{l}-0.07 \\
(0.10)\end{array}$ \\
\hline CHAIN & $\begin{array}{c}-0.05 \\
(0.06)\end{array}$ & $\begin{array}{l}-0.03 \\
(0.03)\end{array}$ & $\begin{array}{l}-0.03 \\
(0.03)\end{array}$ & $\begin{array}{c}0.00 \\
(0.01)\end{array}$ & $\begin{array}{l}-0.05 \\
(0.06)\end{array}$ \\
\hline AUTONOMOUS & $\begin{array}{c}0.06 \\
(0.10)\end{array}$ & $\begin{array}{c}0.03 \\
(0.05)\end{array}$ & $\begin{array}{c}0.04 \\
(0.05)\end{array}$ & $\begin{array}{c}-0.01 \\
(0.02)\end{array}$ & $\begin{array}{c}0.06 \\
(0.10)\end{array}$ \\
\hline PROJECT & $\begin{array}{l}-0.05 \\
(0.10)\end{array}$ & $\begin{array}{l}-0.04 \\
(0.05)\end{array}$ & $\begin{array}{l}-0.04 \\
(0.05)\end{array}$ & $\begin{array}{c}0.00 \\
(0.02)\end{array}$ & $\begin{array}{l}-0.05 \\
(0.10)\end{array}$ \\
\hline ROTATION & $\begin{array}{c}0.12 \\
(0.09)\end{array}$ & $\begin{array}{c}0.08 \\
(0.05)\end{array}$ & $\begin{array}{c}0.08 \\
(0.05)^{*}\end{array}$ & $\begin{array}{c}0.00 \\
(0.01)\end{array}$ & $\begin{array}{c}0.15 \\
(0.09)^{*}\end{array}$ \\
\hline QUALITY & $\begin{array}{l}-0.08 \\
(0.09)\end{array}$ & $\begin{array}{l}-0.03 \\
(0.05)\end{array}$ & $\begin{array}{l}-0.01 \\
(0.05)\end{array}$ & $\begin{array}{l}-0.01 \\
(0.01)\end{array}$ & $\begin{array}{l}-0.03 \\
(0.09)\end{array}$ \\
\hline HIERARCHY & $\begin{array}{l}-0.06 \\
(0.06)\end{array}$ & $\begin{array}{c}-0.07 \\
(0.03)^{* *}\end{array}$ & $\begin{array}{c}-0.05 \\
(0.03)^{*}\end{array}$ & $\begin{array}{l}-0.01 \\
(0.01)\end{array}$ & $\begin{array}{c}-0.13 \\
(0.06)^{* *}\end{array}$ \\
\hline JUST TIME & $\begin{array}{c}0.03 \\
(0.05)\end{array}$ & $\begin{array}{c}0.02 \\
(0.03)\end{array}$ & $\begin{array}{c}0.01 \\
(0.03)\end{array}$ & $\begin{array}{c}0.01 \\
(0.01)\end{array}$ & $\begin{array}{c}0.02 \\
(0.05)\end{array}$ \\
\hline Variation & $\begin{array}{c}0.12 \\
(0.08)\end{array}$ & $\begin{array}{c}0.03 \\
(0.04)\end{array}$ & $\begin{array}{c}0.04 \\
(0.04)\end{array}$ & $\begin{array}{l}-0.01 \\
(0.01)\end{array}$ & $\begin{array}{c}0.05 \\
(0.08)\end{array}$ \\
\hline Tech. change & $\begin{array}{l}-0.13 \\
(0.10)\end{array}$ & $\begin{array}{l}-0.07 \\
(0.06)\end{array}$ & $\begin{array}{l}-0.05 \\
(0.05)\end{array}$ & $\begin{array}{l}-0.03 \\
(0.02)\end{array}$ & $\begin{array}{l}-0.12 \\
(0.10)\end{array}$ \\
\hline Org. change & $\begin{array}{c}0.03 \\
(0.08)\end{array}$ & $\begin{array}{c}0.03 \\
(0.05)\end{array}$ & $\begin{array}{c}0.02 \\
(0.04)\end{array}$ & $\begin{array}{c}0.00 \\
(0.01)\end{array}$ & $\begin{array}{c}0.04 \\
(0.08)\end{array}$ \\
\hline Increasing & $\begin{array}{c}-0.18 \\
(0.12)\end{array}$ & $\begin{array}{c}-0.02 \\
(0.06)\end{array}$ & $\begin{array}{l}-0.09 \\
(0.06)\end{array}$ & $\begin{array}{c}0.07 \\
(0.02)^{* *}\end{array}$ & $\begin{array}{c}-0.09 \\
(0.12)\end{array}$ \\
\hline Decreasing & $\begin{array}{c}0.10 \\
(0.12)\end{array}$ & $\begin{array}{c}0.04 \\
(0.07)\end{array}$ & $\begin{array}{c}0.05 \\
(0.07)\end{array}$ & $\begin{array}{l}-0.01 \\
(0.02)\end{array}$ & $\begin{array}{c}0.10 \\
(0.12)\end{array}$ \\
\hline Hours & $\begin{array}{l}-0.03 \\
(0.12)\end{array}$ & $\begin{array}{l}-0.02 \\
(0.06)\end{array}$ & $\begin{array}{l}-0.05 \\
(0.06)\end{array}$ & $\begin{array}{c}0.03 \\
(0.02)\end{array}$ & $\begin{array}{c}-0.03 \\
(0.12)\end{array}$ \\
\hline P. Employees & $\begin{array}{c}0.56 \\
(0.20)^{* * *}\end{array}$ & $\begin{array}{c}0.07 \\
(0.11)\end{array}$ & $\begin{array}{c}0.19 \\
(0.10)^{*}\end{array}$ & $\begin{array}{c}-0.12 \\
(0.03)^{* * *}\end{array}$ & $\begin{array}{c}0.17 \\
(0.20)\end{array}$ \\
\hline P. Technicians & $\begin{array}{c}-0.55 \\
(0.30)\end{array}$ & $\begin{array}{c}-0.34 \\
(0.16)^{* *}\end{array}$ & $\begin{array}{c}-0.28 \\
(0.16)^{*}\end{array}$ & $\begin{array}{c}-0.06 \\
(0.05)\end{array}$ & $\begin{array}{c}-0.65 \\
(0.30)^{* *}\end{array}$ \\
\hline P. Managers & $\begin{array}{c}0.13 \\
(0.36)\end{array}$ & $\begin{array}{c}0.13 \\
(0.19)\end{array}$ & $\begin{array}{c}0.17 \\
(0.18)\end{array}$ & $\begin{array}{l}-0.05 \\
(0.06)\end{array}$ & $\begin{array}{c}0.20 \\
(0.35)\end{array}$ \\
\hline P. Women & $\begin{array}{c}0.21 \\
(0.19)\end{array}$ & $\begin{array}{c}0.25 \\
(0.10)^{* *}\end{array}$ & $\begin{array}{c}0.27 \\
(0.10)^{* * *}\end{array}$ & $\begin{array}{l}-0.03 \\
(0.03)\end{array}$ & $\begin{array}{c}0.46 \\
(0.18)^{* * *}\end{array}$ \\
\hline P. Contract & $\begin{array}{c}0.38 \\
(0.41)\end{array}$ & $\begin{array}{c}0.14 \\
(0.20)\end{array}$ & $\begin{array}{c}0.09 \\
(0.20)\end{array}$ & $\begin{array}{c}0.05 \\
(0.06)\end{array}$ & $\begin{array}{c}0.19 \\
(0.37)\end{array}$ \\
\hline $\begin{array}{l}\text { Constant } \\
\text { Sectors (10) }\end{array}$ & $\begin{array}{c}0.35 \\
(0.17)^{* *} \\
\text { YES }\end{array}$ & $\begin{array}{c}0.19 \\
(0.09)^{* *} \\
\text { YES }\end{array}$ & $\begin{array}{c}0.18 \\
(0.09)^{* *} \\
\text { YES }\end{array}$ & $\begin{array}{c}0.02 \\
(0.03) \\
\text { YES }\end{array}$ & $\begin{array}{c}0.32 \\
(0.17)^{* *} \\
\text { YES }\end{array}$ \\
\hline $\begin{array}{l}\text { Obs. } \\
R^{2}\end{array}$ & $\begin{array}{l}1333 \\
0.13\end{array}$ & $\begin{array}{l}1384 \\
0.12\end{array}$ & $\begin{array}{l}1384 \\
0.12\end{array}$ & $\begin{array}{l}1384 \\
0.02\end{array}$ & $\begin{array}{l}1384 \\
0.13\end{array}$ \\
\hline
\end{tabular}

() Standard errors.

For Sectors we write in parentheses the number of economic sectors included in the regression.

${ }^{*}$ Significant at $10 \% *^{*}$ Significant at $5 \%{ }^{* * *}$ Significant at $1 \%$.

Source: REPONSE, DMMO and EMMO surveys. 


\section{OLS estimations with incremental organization}

Table 7: Effect of additive clusters on the labor flows, entries, exits, net flows and churning flows. French establishments 1998.

\begin{tabular}{|c|c|c|c|c|c|}
\hline EMPLOYEES & LABOR FLOWS & ENTRIES & EXITS & NET FLOWS & CHURNING \\
\hline \multirow[t]{2}{*}{ TECHNOLOGY } & 0.33 & 0.18 & 0.15 & 0.03 & 0.30 \\
\hline & $(0.20)$ & $(0.11)$ & $(0.10)$ & $(0.04)$ & $(0.19)$ \\
\hline \multirow[t]{2}{*}{ CHAIN } & -0.22 & -0.09 & -0.13 & 0.04 & -0.22 \\
\hline & $(0.20)$ & $(0.11)$ & $(0.09)$ & $(0.04)$ & $(0.18)$ \\
\hline \multirow[t]{2}{*}{ TEAMWORK } & -0.32 & -0.19 & -0.13 & -0.05 & -0.28 \\
\hline & $(0.13)^{* *}$ & $(0.07)^{* * *}$ & $(0.06)^{* *}$ & $(0.02)^{* *}$ & $(0.12)^{* *}$ \\
\hline \multirow[t]{2}{*}{ FLEXIBILITY } & 0.07 & 0.02 & 0.05 & -0.03 & 0.08 \\
\hline & $(0.13)$ & $(0.07)$ & $(0.06)$ & $(0.03)$ & $(0.12)$ \\
\hline \multirow[t]{2}{*}{ QUALITY } & -0.03 & 0.02 & -0.03 & 0.05 & -0.07 \\
\hline & $(0.29)$ & $(0.16)$ & $(0.13)$ & $(0.05)$ & $(0.27)$ \\
\hline \multirow[t]{2}{*}{ Variation } & 0.06 & 0.01 & 0.05 & -0.04 & 0.07 \\
\hline & $(0.26)$ & $(0.14)$ & $(0.12)$ & $(0.05)$ & $(0.24)$ \\
\hline \multirow[t]{2}{*}{ Tech. change } & -0.11 & -0.09 & -0.03 & -0.06 & -0.09 \\
\hline & $(0.33)$ & $(0.18)$ & $(0.15)$ & $(0.06)$ & $(0.30)$ \\
\hline \multirow[t]{2}{*}{ Org. change } & 0.48 & 0.30 & 0.18 & 0.11 & 0.37 \\
\hline & $(0.27)^{*}$ & $(0.15)^{* *}$ & $(0.13)$ & $(0.05)^{* *}$ & $(0.25)$ \\
\hline Increasing & $\begin{array}{l}-0.08 \\
(0.38)\end{array}$ & $\begin{array}{c}-0.04 \\
(0.21)\end{array}$ & $\begin{array}{l}-0.04 \\
(0.18)\end{array}$ & $\begin{array}{c}0.00 \\
(0.07)\end{array}$ & $\begin{array}{l}-0.02 \\
(0.35)\end{array}$ \\
\hline \multirow[t]{2}{*}{ Decreasing } & -0.10 & -0.07 & -0.03 & -0.04 & -0.06 \\
\hline & $(0.39)$ & $(0.22)$ & $(0.18)$ & $(0.07)$ & $(0.37)$ \\
\hline \multirow[t]{2}{*}{ Hours } & -0.26 & -0.14 & -0.13 & -0.01 & -0.21 \\
\hline & $(0.37)$ & $(0.21)$ & $(0.18)$ & $(0.07)$ & $(0.35)$ \\
\hline \multirow[t]{2}{*}{ P. Employees } & -3.76 & -2.04 & -1.73 & -0.31 & $\begin{array}{l}-3.34 \\
-50 * * *\end{array}$ \\
\hline & $(0.63)^{* * *}$ & $(0.34)^{* * *}$ & $(0.29)^{* * *}$ & $(0.12)^{* * *}$ & $(0.58)^{* * *}$ \\
\hline \multirow[t]{2}{*}{ P. Technicians } & -2.08 & -1.21 & -0.88 & -0.33 & -1.87 \\
\hline & $(0.97)^{* *}$ & $(0.53)^{* *}$ & $(0.45)^{* *}$ & $(0.18)^{*}$ & $(0.90)^{* *}$ \\
\hline \multirow[t]{2}{*}{ P. Managers } & -0.25 & -0.20 & -0.04 & -0.16 & 0.04 \\
\hline & $(1.11)$ & $(0.61)$ & $(0.52)$ & $(0.21)$ & $(1.03)$ \\
\hline \multirow[t]{2}{*}{ P. Women } & 1.55 & 0.76 & 0.81 & -0.05 & 1.54 \\
\hline & $(0.58)^{* * *}$ & $(0.32)^{* *}$ & $(0.27)^{* * *}$ & $(0.11)$ & $(0.54)^{* * *}$ \\
\hline \multirow[t]{2}{*}{ P. Contract } & 2.95 & 1.49 & 1.45 & 0.04 & 2.74 \\
\hline & $(1.19)^{* * *}$ & $(0.65)^{* *}$ & $(0.56)^{* * *}$ & $(0.22)$ & $(1.11)^{* *}$ \\
\hline \multirow[t]{2}{*}{ Constant } & 1.08 & 0.61 & 0.48 & 0.13 & 0.86 \\
\hline & $(0.52)^{* *}$ & $(0.28)^{* *}$ & $(0.24)^{* *}$ & $(0.10)$ & $(0.48)^{*}$ \\
\hline Sectors (10) & YES & YES & YES & YES & YES \\
\hline Obs. & 1359 & 1355 & 1355 & 1355 & 1355 \\
\hline$R^{2}$ & 0.07 & 0.07 & 0.08 & 0.01 & 0.07 \\
\hline
\end{tabular}

() Standard errors.

For Sectors we write in parentheses the number of economic sectors included in the regression.

${ }^{*}$ Significant at $10 \%{ }^{* *}$ Significant at $5 \% . * * *$ Significant at $1 \%$.

Source: REPONSE, DMMO and EMMO surveys. 
Table 8: Effect of additive clusters on total labor flows, entries, exits, net flows and churning flows. French establishments 1998.

\begin{tabular}{|c|c|c|c|c|c|}
\hline TOTAL & LABOR FLOWS & ENTRIES & EXITS & NET FLOWS & CHURNING \\
\hline \multirow[t]{2}{*}{ TECHNOLOGY } & 0.05 & 0.04 & 0.02 & 0.02 & 0.07 \\
\hline & $(0.06)$ & $(0.03)$ & $(0.03)$ & $(0.01)^{*}$ & $(0.06)$ \\
\hline \multirow[t]{2}{*}{ CHAIN } & -0.05 & -0.03 & -0.04 & 0.00 & -0.05 \\
\hline & $(0.06)$ & $(0.03)$ & $(0.03)$ & $(0.01)$ & $(0.06)$ \\
\hline \multirow[t]{2}{*}{ TEAMWORK } & -0.03 & -0.04 & -0.03 & -0.01 & -0.07 \\
\hline & $(0.04)$ & $(0.02)^{*}$ & $(0.02)$ & $(0.01)$ & $(0.04)^{*}$ \\
\hline \multirow[t]{2}{*}{ FLEXIBILITY } & 0.05 & 0.03 & 0.02 & 0.01 & 0.05 \\
\hline & $(0.04)$ & $(0.02)$ & $(0.02)$ & $(0.01)$ & $(0.04)$ \\
\hline \multirow[t]{2}{*}{ QUALITY } & -0.09 & -0.03 & -0.02 & -0.01 & -0.04 \\
\hline & $(0.09)$ & $(0.05)$ & $(0.05)$ & $(0.01)$ & $(0.09)$ \\
\hline \multirow[t]{2}{*}{ Variation } & 0.13 & 0.03 & 0.04 & -0.02 & 0.05 \\
\hline & $(0.08)$ & $(0.04)$ & $(0.04)$ & $(0.01)$ & $(0.08)$ \\
\hline \multirow[t]{2}{*}{ Tech. change } & -0.13 & -0.07 & -0.05 & -0.02 & -0.12 \\
\hline & $(0.10)$ & $(0.06)$ & $(0.05)$ & $(0.02)$ & $(0.10)$ \\
\hline \multirow[t]{2}{*}{ Org. change } & 0.03 & 0.03 & 0.03 & 0.00 & 0.04 \\
\hline & $(0.08)$ & $(0.05)$ & $(0.04)$ & $(0.01)$ & $(0.08)$ \\
\hline \multirow[t]{2}{*}{ Increasing } & -0.18 & -0.02 & -0.09 & 0.07 & -0.09 \\
\hline & $(0.12)$ & $(0.06)$ & $(0.06)$ & $(0.02)^{* * *}$ & $(0.12)$ \\
\hline \multirow[t]{2}{*}{ Decreasing } & 0.10 & 0.04 & 0.04 & -0.01 & 0.10 \\
\hline & $(0.12)$ & $(0.07)$ & $(0.07)$ & $(0.02)$ & $(0.12)$ \\
\hline \multirow[t]{2}{*}{ Hours } & -0.03 & -0.02 & -0.05 & 0.03 & -0.05 \\
\hline & $(0.12)$ & $(0.06)$ & $(0.06)$ & $(0.02)$ & $(0.12)$ \\
\hline \multirow[t]{2}{*}{ P. Employees } & 0.57 & 0.08 & 0.20 & -0.12 & 0.19 \\
\hline & $(0.20)^{* * *}$ & $(0.11)$ & $(0.10)^{* *}$ & $(0.03)^{* * *}$ & $(0.20)$ \\
\hline \multirow[t]{2}{*}{ P. Technicians } & -0.49 & -0.30 & -0.24 & -0.06 & -0.58 \\
\hline & $(0.29)^{*}$ & $(0.16)^{*}$ & $(0.16)$ & $(0.05)$ & $(0.30)^{* *}$ \\
\hline \multirow[t]{2}{*}{ P. Managers } & 0.15 & 0.13 & 0.18 & -0.04 & 0.20 \\
\hline & $(0.35)$ & $(0.19)$ & $(0.18)$ & $(0.06)$ & $(0.35)$ \\
\hline \multirow[t]{2}{*}{ P. Women } & 0.22 & 0.25 & 0.28 & -0.03 & 0.47 \\
\hline & $(0.19)$ & $(0.10)^{* * *}$ & $(0.10)^{* * *}$ & $(0.03)$ & $(0.18)^{* * *}$ \\
\hline \multirow[t]{2}{*}{ P. Contract } & 0.40 & 0.16 & 0.11 & 0.05 & 0.23 \\
\hline & $(0.41)$ & $(0.20)$ & $(0.20)$ & $(0.06)$ & $(0.37)$ \\
\hline \multirow[t]{2}{*}{ Constant } & 0.34 & 0.18 & 0.17 & 0.02 & 0.30 \\
\hline & $(0.16)^{* *}$ & $(0.09)^{* *}$ & $(0.09)^{* *}$ & $(0.03)$ & $(0.16)^{*}$ \\
\hline Sectors (10) & YES & YES & YES & YES & YES \\
\hline Obs. & 1333 & 1384 & 1384 & 1384 & 1384 \\
\hline$R^{2}$ & 0.13 & 0.12 & 0.11 & 0.03 & 0.13 \\
\hline
\end{tabular}

() Standard errors.

For Sectors we write in parentheses the number of economic sectors included in the regression. *Significant at $10 \%{ }^{* *}$ Significant at $5 \% .{ }^{* *}$ Significant at $1 \%$.

Source: REPONSE, DMMO and EMMO surveys. 
OLS estimations with multiplicative clusters 
Table 9: Effect of multiplicative clusters on the manual workers' labor flows, entries, exits, net flows and churning flows. French establishments 1998.

\begin{tabular}{|c|c|c|c|c|c|}
\hline MANUAL WORK. & LABOR FLOWS & ENTRIES & EXITS & NET FLOWS & CHURNING \\
\hline COMPUTER & $\begin{array}{c}0.17 \\
(0.24)\end{array}$ & $\begin{array}{c}0.05 \\
(0.12)\end{array}$ & $\begin{array}{c}0.12 \\
(0.13)\end{array}$ & $\begin{array}{l}-0.07 \\
(0.05)\end{array}$ & $\begin{array}{c}0.08 \\
(0.20)\end{array}$ \\
\hline NET & 0.39 & 0.23 & 0.16 & 0.07 & 0.40 \\
\hline & $(0.19)^{* *}$ & $(0.09)^{* *}$ & $(0.10)$ & $(0.04)^{*}$ & $(0.16)^{* *}$ \\
\hline CHAIN & $\begin{array}{c}-0.26 \\
(0.11)^{* *}\end{array}$ & $\begin{array}{c}-0.13 \\
(0.06)^{* *}\end{array}$ & $\begin{array}{c}-0.14 \\
(0.06)^{* *}\end{array}$ & $\begin{array}{c}0.01 \\
(0.03)\end{array}$ & $\begin{array}{c}-0.22 \\
(0.09)^{* *}\end{array}$ \\
\hline AUTONOMOUS & $\begin{array}{l}-0.05 \\
(0.19)\end{array}$ & $\begin{array}{l}-0.03 \\
(0.10)\end{array}$ & $\begin{array}{l}-0.02 \\
(0.10)\end{array}$ & $\begin{array}{l}-0.02 \\
(0.04)\end{array}$ & $\begin{array}{l}-0.06 \\
(0.16)\end{array}$ \\
\hline PROJECT & $\begin{array}{l}-0.31 \\
(0.20)\end{array}$ & $\begin{array}{l}-0.17 \\
(0.10)^{*}\end{array}$ & $\begin{array}{l}-0.14 \\
(0.11)\end{array}$ & $\begin{array}{l}-0.03 \\
(0.05)\end{array}$ & $\begin{array}{l}-0.28 \\
(0.17)^{*}\end{array}$ \\
\hline ROTATION & $\begin{array}{l}-0.07 \\
(0.17)\end{array}$ & $\begin{array}{l}-0.04 \\
(0.09)\end{array}$ & $\begin{array}{l}-0.03 \\
(0.09)\end{array}$ & $\begin{array}{l}-0.02 \\
(0.04)\end{array}$ & $\begin{array}{l}-0.08 \\
(0.15)\end{array}$ \\
\hline QUALITY & $\begin{array}{l}-0.08 \\
(0.17)\end{array}$ & $\begin{array}{l}-0.07 \\
(0.08)\end{array}$ & $\begin{array}{c}0.00 \\
(0.09)\end{array}$ & $\begin{array}{c}-0.07 \\
(0.04)^{* *}\end{array}$ & $\begin{array}{l}-0.08 \\
(0.14)\end{array}$ \\
\hline HIERARCHY & $\begin{array}{c}0.05 \\
(0.11)\end{array}$ & $\begin{array}{c}0.02 \\
(0.05)\end{array}$ & $\begin{array}{c}0.02 \\
(0.06)\end{array}$ & $\begin{array}{c}0.00 \\
(0.02)\end{array}$ & $\begin{array}{c}0.01 \\
(0.09)\end{array}$ \\
\hline JUST TIME & $\begin{array}{c}0.08 \\
(0.09)\end{array}$ & $\begin{array}{c}0.04 \\
(0.04)\end{array}$ & $\begin{array}{c}0.04 \\
(0.05)\end{array}$ & $\begin{array}{c}0.00 \\
(0.02)\end{array}$ & $\begin{array}{c}0.06 \\
(0.07)\end{array}$ \\
\hline TEAMWORK* & $\begin{array}{c}0.08 \\
(0.21)\end{array}$ & $\begin{array}{c}0.05 \\
(0.11)\end{array}$ & $\begin{array}{c}0.02 \\
(0.11)\end{array}$ & $\begin{array}{c}0.03 \\
(0.05)\end{array}$ & $\begin{array}{c}0.09 \\
(0.18)\end{array}$ \\
\hline ICT FLEXIBILITY & $\begin{array}{l}-0.31 \\
(0.40)\end{array}$ & $\begin{array}{l}-0.09 \\
(0.20)\end{array}$ & $\begin{array}{l}-0.22 \\
(0.21)\end{array}$ & $\begin{array}{c}0.12 \\
(0.09)\end{array}$ & $\begin{array}{l}-0.15 \\
(0.34)\end{array}$ \\
\hline Variation & $\begin{array}{c}0.00 \\
(0.15)\end{array}$ & $\begin{array}{l}-0.01 \\
(0.07)\end{array}$ & $\begin{array}{c}0.01 \\
(0.08)\end{array}$ & $\begin{array}{l}-0.03 \\
(0.03)\end{array}$ & $\begin{array}{l}-0.01 \\
(0.12)\end{array}$ \\
\hline Tech. change & $\begin{array}{l}-0.36 \\
(0.19)^{*}\end{array}$ & $\begin{array}{l}-0.18 \\
(0.10)^{*}\end{array}$ & $\begin{array}{l}-0.18 \\
(0.10)^{*}\end{array}$ & $\begin{array}{c}0.01 \\
(0.04)\end{array}$ & $\begin{array}{c}-0.33 \\
(0.16)^{* *}\end{array}$ \\
\hline Org. change & $\begin{array}{l}-0.09 \\
(0.15)\end{array}$ & $\begin{array}{l}-0.03 \\
(0.08)\end{array}$ & $\begin{array}{l}-0.06 \\
(0.08)\end{array}$ & $\begin{array}{c}0.04 \\
(0.03)\end{array}$ & $\begin{array}{l}-0.06 \\
(0.13)\end{array}$ \\
\hline Increasing & $\begin{array}{l}-0.19 \\
(0.22)\end{array}$ & $\begin{array}{l}-0.03 \\
(0.11)\end{array}$ & $\begin{array}{l}-0.15 \\
(0.12)\end{array}$ & $\begin{array}{c}0.12 \\
(0.05)^{* *}\end{array}$ & $\begin{array}{l}-0.15 \\
(0.19)\end{array}$ \\
\hline Decreasing & $\begin{array}{l}-0.22 \\
(0.22)\end{array}$ & $\begin{array}{l}-0.12 \\
(0.11)\end{array}$ & $\begin{array}{l}-0.10 \\
(0.12)\end{array}$ & $\begin{array}{l}-0.02 \\
(0.05)\end{array}$ & $\begin{array}{l}-0.18 \\
(0.18)\end{array}$ \\
\hline Hours & $\begin{array}{l}-0.05 \\
(0.21)\end{array}$ & $\begin{array}{c}0.00 \\
(0.10)\end{array}$ & $\begin{array}{l}-0.05 \\
(0.11)\end{array}$ & $\begin{array}{c}0.04 \\
(0.05)\end{array}$ & $\begin{array}{l}-0.03 \\
(0.17)\end{array}$ \\
\hline P. Employees & $\begin{array}{c}2.90 \\
(0.50)^{* * *}\end{array}$ & $\begin{array}{c}1.48 \\
(0.25)^{* * *}\end{array}$ & $\begin{array}{c}1.41 \\
(0.26)^{* * *}\end{array}$ & $\begin{array}{c}0.07 \\
(0.11)\end{array}$ & $\begin{array}{c}2.44 \\
(0.42)^{* * *}\end{array}$ \\
\hline P. Technicians & $\begin{array}{c}1.01 \\
(0.61)^{*}\end{array}$ & $\begin{array}{c}0.51 \\
(0.30)^{*}\end{array}$ & $\begin{array}{c}0.49 \\
(0.33)\end{array}$ & $\begin{array}{c}0.01 \\
(0.14)\end{array}$ & $\begin{array}{c}0.63 \\
(0.52)\end{array}$ \\
\hline P. Managers & $\begin{array}{c}0.18 \\
(0.80)\end{array}$ & $\begin{array}{l}-0.23 \\
(0.40)\end{array}$ & $\begin{array}{c}0.42 \\
(0.43)\end{array}$ & $\begin{array}{c}-0.65 \\
(0.18)^{* * *}\end{array}$ & $\begin{array}{l}-0.29 \\
(0.68)\end{array}$ \\
\hline P. Women & $\begin{array}{c}0.57 \\
(0.34)^{*}\end{array}$ & $\begin{array}{c}0.22 \\
(0.17)\end{array}$ & $\begin{array}{c}0.37 \\
(0.18)^{* *}\end{array}$ & $\begin{array}{c}-0.15 \\
(0.08)^{* *}\end{array}$ & $\begin{array}{c}0.37 \\
(0.29)\end{array}$ \\
\hline P. Contract & $\begin{array}{c}1.10 \\
(0.68)\end{array}$ & $\begin{array}{c}0.54 \\
(0.34)\end{array}$ & $\begin{array}{c}0.56 \\
(0.36)\end{array}$ & $\begin{array}{l}-0.02 \\
(0.15)\end{array}$ & $\begin{array}{c}1.01 \\
(0.58)^{*}\end{array}$ \\
\hline Constant & $\begin{array}{c}0.14 \\
(0.30) \\
\text { YES }\end{array}$ & $\begin{array}{c}0.13 \\
(0.15) \\
\text { YES }\end{array}$ & $\begin{array}{c}0.02 \\
(0.16)\end{array}$ & $\begin{array}{c}0.11 \\
(0.07) \\
\text { YES }\end{array}$ & $\begin{array}{c}0.26 \\
(0.25)\end{array}$ \\
\hline $\begin{array}{l}\text { Sectors (10) } \\
\text { Obs. }\end{array}$ & $\begin{array}{c}\text { YES } \\
1079\end{array}$ & $\begin{array}{l}\text { YES } \\
1076\end{array}$ & $\begin{array}{l}\text { YES } \\
1076\end{array}$ & $\begin{array}{l}\text { YES } \\
1076\end{array}$ & $\begin{array}{l}\text { YES } \\
1076\end{array}$ \\
\hline$R^{2}$ & 0.09 & 0.08 & 0.09 & 0.06 & 0.08 \\
\hline
\end{tabular}

() Standard errors.

For Sectors we write in parentheses the number of economic sectors included in the regression.

*Significant at $10 \%$.**Significant at $5 \%{ }^{* * *}$ Significant at $1 \%$.

Source: REPONSE, DMMO and EMMO surveys. 
Table 10: Effect of multiplicative clusters on the total labor flows, entries, exits, net flows and churning flows. French establishments 1998.

\begin{tabular}{|c|c|c|c|c|c|}
\hline TOTAL & LABOR FLOWS & ENTRIES & EXITS & NET FLOWS & CHURNING \\
\hline COMPUTER & $\begin{array}{c}0.07 \\
(0.12)\end{array}$ & $\begin{array}{c}0.06 \\
(0.07)\end{array}$ & $\begin{array}{c}0.06 \\
(0.07)\end{array}$ & $\begin{array}{c}0.00 \\
(0.02)\end{array}$ & $\begin{array}{c}0.07 \\
(0.13)\end{array}$ \\
\hline NET & $\begin{array}{l}-0.11 \\
(0.10)\end{array}$ & $\begin{array}{l}-0.05 \\
(0.06)\end{array}$ & $\begin{array}{l}-0.07 \\
(0.05)\end{array}$ & $\begin{array}{c}0.03 \\
(0.02)\end{array}$ & $\begin{array}{l}-0.07 \\
(0.10)\end{array}$ \\
\hline CHAIN & $\begin{array}{l}-0.05 \\
(0.06)\end{array}$ & $\begin{array}{l}-0.03 \\
(0.03)\end{array}$ & $\begin{array}{l}-0.03 \\
(0.03)\end{array}$ & $\begin{array}{c}0.00 \\
(0.01)\end{array}$ & $\begin{array}{l}-0.05 \\
(0.06)\end{array}$ \\
\hline AUTONOMOUS & $\begin{array}{l}-0.01 \\
(0.11)\end{array}$ & $\begin{array}{c}0.00 \\
(0.06)\end{array}$ & $\begin{array}{c}0.02 \\
(0.06)\end{array}$ & $\begin{array}{l}-0.01 \\
(0.02)\end{array}$ & $\begin{array}{c}0.01 \\
(0.11)\end{array}$ \\
\hline PROJECT & $\begin{array}{l}-0.12 \\
(0.11)\end{array}$ & $\begin{array}{l}-0.06 \\
(0.06)\end{array}$ & $\begin{array}{l}-0.06 \\
(0.06)\end{array}$ & $\begin{array}{c}0.00 \\
(0.02)\end{array}$ & $\begin{array}{l}-0.10 \\
(0.11)\end{array}$ \\
\hline ROTATION & $\begin{array}{l}-0.03 \\
(0.10)\end{array}$ & $\begin{array}{c}0.00 \\
(0.06)\end{array}$ & $\begin{array}{c}0.01 \\
(0.05)\end{array}$ & $\begin{array}{l}-0.01 \\
(0.02)\end{array}$ & $\begin{array}{c}0.01 \\
(0.10)\end{array}$ \\
\hline QUALITY & $\begin{array}{l}-0.08 \\
(0.09)\end{array}$ & $\begin{array}{l}-0.03 \\
(0.05)\end{array}$ & $\begin{array}{l}-0.01 \\
(0.05)\end{array}$ & $\begin{array}{l}-0.01 \\
(0.01)\end{array}$ & $\begin{array}{l}-0.03 \\
(0.09)\end{array}$ \\
\hline HIERARCHY & $\begin{array}{l}-0.07 \\
(0.06)\end{array}$ & $\begin{array}{c}-0.07 \\
(0.03)^{* *}\end{array}$ & $\begin{array}{l}-0.06 \\
(0.03)^{*}\end{array}$ & $\begin{array}{l}-0.01 \\
(0.01)\end{array}$ & $\begin{array}{c}-0.14 \\
(0.06)^{* *}\end{array}$ \\
\hline JUST TIME & $\begin{array}{c}0.02 \\
(0.05)\end{array}$ & $\begin{array}{c}0.01 \\
(0.03)\end{array}$ & $\begin{array}{c}0.00 \\
(0.03)\end{array}$ & $\begin{array}{c}0.01 \\
(0.01)\end{array}$ & $\begin{array}{c}0.01 \\
(0.05)\end{array}$ \\
\hline TEAMWORK* & $\begin{array}{c}0.16 \\
(0.12)\end{array}$ & $\begin{array}{c}0.05 \\
(0.07)\end{array}$ & $\begin{array}{c}0.05 \\
(0.06)\end{array}$ & $\begin{array}{c}0.00 \\
(0.02)\end{array}$ & $\begin{array}{c}0.10 \\
(0.12)\end{array}$ \\
\hline ICT FLEXIBILITY & $\begin{array}{c}0.69 \\
(0.21)^{* * *}\end{array}$ & $\begin{array}{c}0.36 \\
(0.12)^{* * *}\end{array}$ & $\begin{array}{c}0.31 \\
(0.11)^{* * *}\end{array}$ & $\begin{array}{c}0.05 \\
(0.03)\end{array}$ & $\begin{array}{c}0.69 \\
(0.21)^{* * *}\end{array}$ \\
\hline Variation & $\begin{array}{c}0.13 \\
(0.08)\end{array}$ & $\begin{array}{c}0.03 \\
(0.04)\end{array}$ & $\begin{array}{c}0.04 \\
(0.04)\end{array}$ & $\begin{array}{l}-0.01 \\
(0.01)\end{array}$ & $\begin{array}{c}0.06 \\
(0.08)\end{array}$ \\
\hline Tech. change & $\begin{array}{l}-0.14 \\
(0.10)\end{array}$ & $\begin{array}{l}-0.08 \\
(0.06)\end{array}$ & $\begin{array}{l}-0.05 \\
(0.05)\end{array}$ & $\begin{array}{l}-0.03 \\
(0.02)\end{array}$ & $\begin{array}{l}-0.13 \\
(0.10)\end{array}$ \\
\hline Org. change & $\begin{array}{c}0.01 \\
(0.08)\end{array}$ & $\begin{array}{c}0.02 \\
(0.05)\end{array}$ & $\begin{array}{c}0.01 \\
(0.04)\end{array}$ & $\begin{array}{c}0.00 \\
(0.01)\end{array}$ & $\begin{array}{c}0.02 \\
(0.08)\end{array}$ \\
\hline Increasing & $\begin{array}{l}-0.18 \\
(0.12)\end{array}$ & $\begin{array}{l}-0.02 \\
(0.06)\end{array}$ & $\begin{array}{l}-0.09 \\
(0.06)\end{array}$ & $\begin{array}{c}0.07 \\
(0.02)^{* * *}\end{array}$ & $\begin{array}{l}-0.09 \\
(0.12)\end{array}$ \\
\hline Decreasing & $\begin{array}{c}0.09 \\
(0.12)\end{array}$ & $\begin{array}{c}0.04 \\
(0.07)\end{array}$ & $\begin{array}{c}0.05 \\
(0.06)\end{array}$ & $\begin{array}{c}-0.01 \\
(0.02)\end{array}$ & $\begin{array}{c}0.11 \\
(0.12)\end{array}$ \\
\hline Hours & $\begin{array}{l}-0.03 \\
(0.12)\end{array}$ & $\begin{array}{l}-0.02 \\
(0.06)\end{array}$ & $\begin{array}{l}-0.05 \\
(0.06)\end{array}$ & $\begin{array}{c}0.03 \\
(0.02)\end{array}$ & $\begin{array}{l}-0.04 \\
(0.12)\end{array}$ \\
\hline P. Employees & $\begin{array}{c}0.53 \\
(0.20)^{* * *}\end{array}$ & $\begin{array}{c}0.05 \\
(0.11)\end{array}$ & $\begin{array}{c}0.17 \\
(0.10)^{*}\end{array}$ & $\begin{array}{c}-0.12 \\
(0.03)^{* * *}\end{array}$ & $\begin{array}{c}0.14 \\
(0.20)\end{array}$ \\
\hline P. Technicians & $\begin{array}{c}-0.56 \\
(0.29)^{*}\end{array}$ & $\begin{array}{c}-0.36 \\
(0.16)^{* *}\end{array}$ & $\begin{array}{l}-0.30 \\
(0.16)^{*}\end{array}$ & $\begin{array}{l}-0.06 \\
(0.05)\end{array}$ & $\begin{array}{c}-0.69 \\
(0.30)^{* *}\end{array}$ \\
\hline P. Managers & $\begin{array}{c}0.27 \\
(0.36)\end{array}$ & $\begin{array}{c}0.20 \\
(0.19)\end{array}$ & $\begin{array}{c}0.23 \\
(0.19)\end{array}$ & $\begin{array}{l}-0.04 \\
(0.06)\end{array}$ & $\begin{array}{c}0.34 \\
(0.35)\end{array}$ \\
\hline P. Women & $\begin{array}{c}0.23 \\
(0.18)\end{array}$ & $\begin{array}{c}0.26 \\
(0.10)^{* * *}\end{array}$ & $\begin{array}{c}0.29 \\
(0.10)^{* * *}\end{array}$ & $\begin{array}{l}-0.02 \\
(0.03)\end{array}$ & $\begin{array}{c}0.49 \\
(0.18)^{* * *}\end{array}$ \\
\hline P. Contract & $\begin{array}{c}0.34 \\
(0.41)\end{array}$ & $\begin{array}{c}0.12 \\
(0.20)\end{array}$ & $\begin{array}{c}0.08 \\
(0.20)\end{array}$ & $\begin{array}{c}0.05 \\
(0.06)\end{array}$ & $\begin{array}{c}0.16 \\
(0.37)\end{array}$ \\
\hline $\begin{array}{l}\text { Constant } \\
\text { Sectors (10) }\end{array}$ & $\begin{array}{c}0.43 \\
(0.17)^{* * *} \\
\text { YES }\end{array}$ & $\begin{array}{c}0.23 \\
(0.09)^{* * *} \\
\text { YES }\end{array}$ & $\begin{array}{c}0.20 \\
(0.09)^{* * *} \\
\text { YES }\end{array}$ & $\begin{array}{c}0.02 \\
(0.03) \\
\text { YES }\end{array}$ & $\begin{array}{c}0.38 \\
(0.17)^{* *} \\
\text { YES }\end{array}$ \\
\hline Obs. & 1333 & 1384 & 1384 & 1384 & 1384 \\
\hline$R^{2}$ & 0.14 & 0.13 & 0.12 & 0.02 & 0.13 \\
\hline
\end{tabular}

() Standard errors.

For Sectors we write in parentheses the number of economic sectors included in the regression.

*Significant at $10 \%{ }^{* *}$ Significant at $5 \%{ }^{* * *}$ Significant at $1 \%$.

Source: REPONSE, DMMO and EMMO surveys. 


\section{Appendix B: Descriptive statistics}

\section{Means and standard deviations}

Table 11: Mean and standard deviations of gross labor flows, entries, exits, net labor flows and churning flows in cross sectional analysis. French establishments 1998.

\begin{tabular}{|l|ccc|}
\hline Variables & Observations & Mean & Standard Deviations \\
\hline MANAGERS & 1825 & 0.764 & 18.800 \\
GROSS LABOR FLOWS & 1818 & 0.372 & 9.407 \\
ENTRIES & 1818 & 0.394 & 9.431 \\
EXITS & 1818 & -0.022 & 0.238 \\
NET LABOR FLOWS & 1818 & 0.669 & 18.810 \\
CHURNING FLOWS & & & \\
\hline INTERMEDIATE PROFESSIONS & 1720 & 0.697 & 2.835 \\
GROSS LABOR FLOWS & 1680 & 0.359 & 1.482 \\
ENTRIES & 1680 & 0.354 & 1.417 \\
EXITS & 1680 & 0.005 & 0.435 \\
NET LABOR FLOWS & 1680 & 0.566 & 2.734 \\
CHURNING FLOWS & & & \\
\hline EMPLOYEES & 1803 & 0.921 & 4.323 \\
GROSS LABOR FLOWS & 1756 & 0.503 & 2.506 \\
ENTRIES & 1756 & 0.443 & 2.007 \\
EXITS & 1756 & 0.059 & 1.205 \\
NET LABOR FLOWS & 1756 & 0.795 & 3.988 \\
CHURNING FLOWS & & & \\
\hline MANUAL WORKERS & 1378 & 0.574 & 2.149 \\
GROSS LABOR FLOWS & 1374 & 0.277 & 1.052 \\
ENTRIES & 1374 & 0.299 & 1.156 \\
EXITS & 1374 & -0.022 & 0.502 \\
NET LABOR FLOWS & 1374 & 0.454 & 1.807 \\
CHURNING FLOWS & & & \\
\hline TOTAL & 2024 & 0.741 & 2.213 \\
GROSS LABOR FLOWS & 2119 & 0.375 & 1.017 \\
ENTRIES & 2119 & 0.365 & 0.897 \\
EXITS & 2119 & 0.010 & 0.404 \\
NET LABOR FLOWS & 2119 & 0.649 & 1.734 \\
CHURNING FLOWS & & & \\
\hline
\end{tabular}

Source: REPONSE, DMMO and EMMO surveys. 


\section{Means and standard deviations}

Table 12: Mean and standard deviations of explicative and control variables in cross sectional analysis. French establishments 1998.

\begin{tabular}{|l|ccc|}
\hline Variables & Observations & Mean & Standard Deviations \\
\hline COMPUTER & 2841 & 0.317 & 0.465 \\
NET & 2844 & 0.252 & 0.435 \\
INTERNET & 2844 & 0.069 & 0.254 \\
CHAIN & 2828 & 0.780 & 0.806 \\
AUTONOMOUS & 2844 & 0.192 & 0.394 \\
PROJECT & 2844 & 0.244 & 0.430 \\
ROTATION & 2833 & 0.252 & 0.434 \\
QUALITY & 2831 & 0.609 & 0.488 \\
HIERARCHY & 2789 & 0.931 & 0.728 \\
J.I.T. & 2760 & 0.722 & 0.852 \\
\hline Union & 2844 & 0.392 & 0.488 \\
Variation & 2821 & 0.424 & 0.494 \\
Tech. change & 2837 & 0.185 & 0.388 \\
Org. change & 2843 & 0.350 & 0.477 \\
Strongly increasing & 2818 & 0.133 & 0.339 \\
Increasing & 2818 & 0.426 & 0.495 \\
Decreasing & 2818 & 0.118 & 0.322 \\
Strongly decreasing & 2818 & 0.022 & 0.147 \\
Size 20-50 & 2844 & 0.252 & 0.434 \\
Size +500 & 2844 & 0.124 & 0.329 \\
Hours & 2767 & 0.109 & 0.312 \\
P. Employees & 2430 & 0.362 & 1.453 \\
P. Technicians & 2252 & 0.270 & 2.593 \\
P. Managers & 2456 & 0.127 & 0.152 \\
P. Women & 2649 & 0.380 & 0.285 \\
P. Contract & 2628 & 0.058 & 0.114 \\
\hline & & &
\end{tabular}

Source: REPONSE, DMMO and EMMO surveys. 


\section{Correlation matrix}

Table 13: Correlation matrix. French establishments 1998.

\begin{tabular}{|c|c|c|c|c|c|c|c|}
\hline & COMPUTER & NET & CHAIN & AUTONOMOUS & PROJECT & ROTATION & QUALITY \\
\hline COMPUTER & \multirow{10}{*}{$\begin{array}{c}1.000 \\
0.496 \\
(0.000) \\
-0.012 \\
(0.531) \\
-0.038 \\
(0.042) \\
0.184 \\
(0.000) \\
-0.107 \\
(0.000) \\
-0.004 \\
(0.841) \\
0.075 \\
(0.000) \\
-0.081 \\
(0.000) \\
\end{array}$} & & & & & & \\
\hline NET & & 1.000 & & & & & \\
\hline CHAIN & & 0.086 & 1.000 & & & & \\
\hline AUTONOMOUS & & $\begin{array}{l}(0.000) \\
0.036)\end{array}$ & 0.171 & 1.000 & & & \\
\hline PROJECT & & $\begin{array}{c}(0.056) \\
0.204\end{array}$ & $\begin{array}{c}(0.000) \\
0.061\end{array}$ & 0.153 & 1.000 & & \\
\hline ROTATION & & $\begin{array}{l}(0.000) \\
-0.049\end{array}$ & $\begin{array}{c}(0.001) \\
0.134\end{array}$ & $\begin{array}{c}(0.000) \\
0.098\end{array}$ & -0.004 & 1.000 & \\
\hline QUALITY & & $\begin{array}{c}(0.010) \\
0.061\end{array}$ & $\begin{array}{c}(0.000) \\
0.317\end{array}$ & $\begin{array}{c}(0.000) \\
0.159\end{array}$ & $\begin{array}{c}(0.822) \\
0.100\end{array}$ & 0.095 & 1.000 \\
\hline \multirow{4}{*}{$\begin{array}{l}\text { HIERARCHY } \\
\text { J.I.T. }\end{array}$} & & $(0.001)$ & $(0.000)$ & $(0.000)$ & $(0.000)$ & $(0.000)$ & \\
\hline & & 0.136 & 0.249 & $\begin{array}{l}0.117 \\
(0.000)\end{array}$ & $\begin{array}{l}0.144 \\
(0.000)\end{array}$ & 0.061 & $\begin{array}{l}0.209 \\
(0.000)\end{array}$ \\
\hline & & $\begin{array}{c}-0.002 \\
(0.931)\end{array}$ & $\begin{array}{c}0.316 \\
(0.000)\end{array}$ & $\begin{array}{c}0.135 \\
(0.000)\end{array}$ & $\begin{array}{c}0.007 \\
(0.696)\end{array}$ & $\begin{array}{c}0.169 \\
(0.000)\end{array}$ & $\begin{array}{c}(0.042) \\
0.242 \\
0.000)\end{array}$ \\
\hline & HIERARCHY & & & & & & \\
\hline HIERARCHY & 1.000 & & & & & & \\
\hline J.I.T. & $\begin{array}{c}0.210 \\
(0.000)\end{array}$ & 1.000 & & & & & \\
\hline
\end{tabular}

() Significance levels

Source: REPONSE, DMMO and EMMO surveys. 
8 Complements for the Referees: other detailed tables

OLS estimations 
Table 14: Determinants of the intermediary professionals' labor flows, entries, exits, net flows and churning flows. French establishments 1998.

\begin{tabular}{|c|c|c|c|c|c|}
\hline INTERMED. PROF. & LABOR FLOWS & ENTRIES & EXITS & NET FLOWS & CHURNING \\
\hline \multirow[t]{2}{*}{ COMPUTER } & 0.23 & 0.14 & 0.10 & 0.04 & 0.18 \\
\hline & $(0.23)$ & $(0.12)$ & $(0.11)$ & $(0.04)$ & $(0.22)$ \\
\hline \multirow[t]{2}{*}{ NET } & -0.01 & -0.02 & 0.00 & -0.02 & 0.03 \\
\hline & $(0.21)$ & $(0.11)$ & $(0.10)$ & $(0.03)$ & $(0.20)$ \\
\hline \multirow[t]{2}{*}{ CHAIN } & 0.06 & 0.04 & 0.02 & 0.02 & 0.09 \\
\hline & $(0.12)$ & $(0.06)$ & $(0.06)$ & $(0.02)$ & $(0.12)$ \\
\hline \multirow[t]{2}{*}{ AUTONOMOUS } & 0.14 & 0.05 & 0.08 & -0.03 & 0.14 \\
\hline & $(0.20)$ & $(0.10)$ & $(0.10)$ & $(0.03)$ & $(0.19)$ \\
\hline \multirow[t]{2}{*}{ PROJECT } & -0.28 & -0.14 & -0.14 & 0.00 & -0.27 \\
\hline & $(0.19)$ & $(0.10)$ & $(0.10)$ & $(0.03)$ & $(0.19)$ \\
\hline \multirow[t]{2}{*}{ ROTATION } & 0.18 & 0.09 & 0.09 & 0.00 & 0.14 \\
\hline & $(0.18)$ & $(0.09)$ & $(0.09)$ & $(0.03)$ & $(0.18)$ \\
\hline \multirow[t]{2}{*}{ QUALITY } & 0.18 & 0.06 & 0.12 & -0.06 & 0.18 \\
\hline & $(0.18)$ & $(0.09)$ & $(0.09)$ & $(0.03)^{* *}$ & $(0.17)$ \\
\hline \multirow[t]{2}{*}{ HIERARCHY } & -0.20 & -0.11 & -0.09 & -0.01 & -0.20 \\
\hline & $(0.12)^{*}$ & $(0.06)^{*}$ & $(0.06)$ & $(0.02)$ & $(0.11)^{*}$ \\
\hline \multirow[t]{2}{*}{ JUST TIME } & -0.04 & -0.02 & -0.02 & 0.00 & -0.04 \\
\hline & $(0.10)$ & $(0.05)$ & $(0.05)$ & $(0.02)$ & $(0.09)$ \\
\hline \multirow[t]{2}{*}{ Variation } & 0.13 & 0.03 & 0.09 & -0.06 & 0.10 \\
\hline & $(0.16)$ & $(0.08)$ & $(0.08)$ & $(0.03)^{* *}$ & $(0.16)$ \\
\hline \multirow[t]{2}{*}{ Tech. change } & 0.13 & 0.06 & 0.08 & -0.02 & 0.17 \\
\hline & $(0.20)$ & $(0.11)$ & $(0.10)$ & $(0.03)$ & $(0.20)$ \\
\hline \multirow[t]{2}{*}{ Org. change } & 0.00 & 0.00 & 0.00 & -0.01 & -0.06 \\
\hline & $(0.17)$ & $(0.09)$ & $(0.08)$ & $(0.03)$ & $(0.16)$ \\
\hline \multirow[t]{2}{*}{ Increasing } & -0.23 & -0.08 & -0.16 & 0.08 & -0.18 \\
\hline & $(0.24)$ & $(0.12)$ & $(0.12)$ & $(0.04)^{* *}$ & $(0.23)$ \\
\hline \multirow[t]{2}{*}{ Decreasing } & 0.44 & 0.20 & 0.23 & -0.03 & 0.47 \\
\hline & $(0.25)^{*}$ & $(0.13)$ & $(0.12)^{*}$ & $(0.04)$ & $(0.24)^{* *}$ \\
\hline \multirow[t]{2}{*}{ Hours } & 0.26 & 0.14 & 0.13 & 0.01 & 0.23 \\
\hline & $(0.23)$ & $(0.12)$ & $(0.12)$ & $(0.04)$ & $(0.22)$ \\
\hline \multirow[t]{2}{*}{ P. Employees } & 0.72 & 0.38 & 0.35 & 0.03 & 0.57 \\
\hline & $(0.39)^{*}$ & $(0.20)^{*}$ & $(0.20)^{*}$ & $(0.07)$ & $(0.38)$ \\
\hline \multirow[t]{2}{*}{ P. Technicians } & -3.21 & -1.64 & -1.58 & -0.06 & -2.63 \\
\hline & $(0.60)^{* * *}$ & $(0.31)^{* * *}$ & $(0.30)^{* * *}$ & $(0.10)$ & $(0.57)^{* * *}$ \\
\hline \multirow[t]{2}{*}{ P. Managers } & 3.25 & 1.74 & 1.53 & 0.21 & 2.90 \\
\hline & $(0.70)^{* * *}$ & $(0.36)^{* * *}$ & $(0.35)^{* * *}$ & $(0.12)^{*}$ & $(0.67)^{* * *}$ \\
\hline \multirow[t]{2}{*}{ P. Women } & 0.34 & 0.12 & 0.21 & -0.09 & 0.37 \\
\hline & $(0.36)$ & $(0.19)$ & $(0.18)$ & $(0.06)$ & $(0.35)$ \\
\hline \multirow[t]{2}{*}{ P. Contract } & 0.74 & 0.45 & 0.29 & 0.16 & 0.68 \\
\hline & $(0.74)$ & $(0.38)$ & $(0.37)$ & $(0.12)$ & $(0.71)$ \\
\hline \multirow[t]{2}{*}{ Constant } & 0.07 & 0.06 & 0.02 & 0.05 & -0.10 \\
\hline & $(0.33)$ & $(0.17)$ & $(0.16)$ & $(0.05)$ & $(0.32)$ \\
\hline Sectors (10) & YES & YES & YES & YES & YES \\
\hline Obs. & 1374 & 1371 & 1371 & 1371 & 1371 \\
\hline$R^{2}$ & 0.15 & 0.15 & 0.14 & 0.02 & 0.14 \\
\hline
\end{tabular}

() Standard errors.

For Sectors we write in parentheses the number of economic sectors included in the regression.

*Significant at $10 \%{ }^{* *}$ Significant at $5 \% . * * *$ Significant at $1 \%$.

Source: REPONSE, DMMO and EMMO surveys. 
Table 15: Determinants of the employees' labor flows, entries, exits, net flows and churning flows. French establishments 1998.

\begin{tabular}{|c|c|c|c|c|c|}
\hline EMPLOYEES & LABOR FLOWS & ENTRIES & EXITS & NET FLOWS & CHURNING \\
\hline \multirow[t]{2}{*}{ COMPUTER } & 0.78 & 0.41 & 0.36 & 0.05 & 0.74 \\
\hline & $(0.36)^{* *}$ & $(0.20)^{* *}$ & $(0.17)^{* *}$ & $(0.07)$ & $(0.34)^{* *}$ \\
\hline NET & $\begin{array}{c}-0.06 \\
(0.33)\end{array}$ & -0.03 & $\begin{array}{l}-0.03 \\
(0.15)\end{array}$ & $\begin{array}{c}0.00 \\
(0.06)\end{array}$ & $\begin{array}{l}-0.07 \\
(0.31)\end{array}$ \\
\hline \multirow[t]{2}{*}{ CHAIN } & -0.20 & -0.08 & -0.12 & 0.04 & -0.20 \\
\hline & $(0.20)$ & $(0.11)$ & $(0.09)$ & $(0.04)$ & $(0.18)$ \\
\hline \multirow[t]{2}{*}{ AUTONOMOUS } & -0.17 & -0.12 & -0.05 & -0.07 & -0.15 \\
\hline & $(0.32)$ & $(0.17)$ & $(0.15)$ & $(0.06)$ & $(0.29)$ \\
\hline \multirow[t]{2}{*}{ PROJECT } & -0.20 & -0.10 & -0.10 & -0.01 & -0.20 \\
\hline & $(0.31)$ & $(0.17)$ & $(0.15)$ & $(0.06)$ & $(0.29)$ \\
\hline \multirow[t]{2}{*}{ ROTATION } & 0.40 & 0.18 & 0.21 & -0.03 & 0.41 \\
\hline & $(0.29)$ & $(0.16)$ & $(0.14)$ & $(0.06)$ & $(0.27)$ \\
\hline \multirow[t]{2}{*}{ QUALITY } & 0.00 & 0.03 & -0.02 & 0.05 & -0.04 \\
\hline & $(0.29)$ & $(0.16)$ & $(0.13)$ & $(0.05)$ & $(0.27)$ \\
\hline \multirow[t]{2}{*}{ HIERARCHY } & -0.41 & -0.24 & -0.18 & -0.06 & -0.36 \\
\hline & $(0.19)^{* *}$ & $(0.10)^{* *}$ & $(0.09)^{* *}$ & $(0.04)^{*}$ & $(0.17)^{* *}$ \\
\hline JUST TIME & $\begin{array}{l}-0.02 \\
(0.16)\end{array}$ & $\begin{array}{l}-0.03 \\
(0.09)\end{array}$ & 0.00 & -0.02 & $\begin{array}{l}-0.01 \\
(0.15)\end{array}$ \\
\hline \multirow[t]{2}{*}{ Variation } & 0.06 & 0.01 & 0.05 & -0.04 & 0.06 \\
\hline & $(0.26)$ & $(0.14)$ & $(0.12)$ & $(0.05)$ & $(0.24)$ \\
\hline \multirow[t]{2}{*}{ Tech. change } & -0.12 & -0.09 & -0.03 & -0.06 & -0.10 \\
\hline & $(0.33)$ & $(0.18)$ & $(0.15)$ & $(0.06)$ & $(0.30)$ \\
\hline \multirow[t]{2}{*}{ Org. change } & 0.48 & 0.30 & 0.18 & 0.12 & 0.37 \\
\hline & $(0.27)^{*}$ & $(0.15)^{* *}$ & $(0.13)$ & $(0.05)^{* *}$ & $(0.25)$ \\
\hline \multirow[t]{2}{*}{ Increasing } & -0.09 & -0.04 & -0.04 & 0.00 & -0.03 \\
\hline & $(0.38)$ & $(0.21)$ & $(0.18)$ & $(0.07)$ & $(0.35)$ \\
\hline \multirow[t]{2}{*}{ Decreasing } & -0.09 & -0.07 & -0.03 & -0.04 & -0.06 \\
\hline & $(0.40)$ & $(0.22)$ & $(0.19)$ & $(0.07)$ & $(0.37)$ \\
\hline \multirow[t]{2}{*}{ Hours } & -0.24 & -0.13 & -0.12 & -0.01 & -0.19 \\
\hline & $(0.38)$ & $(0.21)$ & $(0.18)$ & $(0.07)$ & $(0.35)$ \\
\hline \multirow[t]{2}{*}{ P. Employees } & -3.82 & -2.07 & -1.76 & -0.32 & -3.39 \\
\hline & $(0.63)^{* * *}$ & $(0.34)^{* * *}$ & $(0.29)^{* * *}$ & $(0.12)^{* * *}$ & $(0.59)^{* * *}$ \\
\hline \multirow[t]{2}{*}{ P. Technicians } & -2.28 & -1.32 & -0.97 & -0.35 & -2.06 \\
\hline & $(0.98) * *$ & $(0.53) * *$ & $(0.46)^{* *}$ & $(0.18)^{*}$ & $(0.91)^{* *}$ \\
\hline P. Managers & -0.32 & -0.26 & -0.06 & -0.20 & $\begin{array}{l}-0.03 \\
(1.06)\end{array}$ \\
\hline \multirow[t]{2}{*}{ P. Women } & $\begin{array}{l}(1.13) \\
1.53\end{array}$ & 0.75 & $\begin{array}{c}(0.53) \\
0.80\end{array}$ & $\begin{array}{l}(0.21) \\
-0.05\end{array}$ & $\begin{array}{c}(1.06) \\
1.51\end{array}$ \\
\hline & $(0.58)^{* * *}$ & $(0.32)^{* *}$ & $(0.27)^{* * *}$ & $(0.11)$ & $(0.54)^{* * *}$ \\
\hline P. Contract & $\begin{array}{l}2.87 \\
(1.19)^{* *}\end{array}$ & $\begin{array}{l}1.45 \\
(0.65) * *\end{array}$ & $\begin{array}{l}1.42 \\
(0.56)^{* * *}\end{array}$ & 0.04 & 2.67 \\
\hline \multirow{2}{*}{ Constant } & $\begin{array}{c}(1.19)^{* *} \\
1.11\end{array}$ & $\begin{array}{c}(0.65)^{* *} \\
0.63\end{array}$ & $\begin{array}{c}(0.56)^{* * *} \\
0.49\end{array}$ & $\begin{array}{c}(0.22) \\
0.15\end{array}$ & $\begin{array}{c}(1.11)^{* *} \\
0.88\end{array}$ \\
\hline & $(0.52)^{* *}$ & $(0.29)^{* *}$ & $(0.25)^{* *}$ & $(0.10)$ & $(0.49)^{*}$ \\
\hline Sectors $(10)$ & YES & YES & YES & YES & YES \\
\hline Obs. & 1359 & 1355 & 1355 & 1355 & 1355 \\
\hline$R^{2}$ & 0.07 & 0.07 & 0.08 & 0.01 & 0.08 \\
\hline
\end{tabular}

() Standard errors.

For Sectors we write in parentheses the number of economic sectors included in the regression.

${ }^{*}$ Significant at $10 \%{ }^{* *}$ Significant at $5 \% . * * *$ Significant at $1 \%$.

Source: REPONSE, DMMO and EMMO surveys. 
Table 16: Determinants of the manual workers' labor flows, entries, exits, net flows and churning flows. French establishments 1998.

\begin{tabular}{|c|c|c|c|c|c|}
\hline MANUAL WORK. & LABOR FLOWS & ENTRIES & EXITS & NET FLOWS & CHURNING \\
\hline COMPUTER & $\begin{array}{c}0.08 \\
(0.21)\end{array}$ & $\begin{array}{c}0.02 \\
(0.10)\end{array}$ & $\begin{array}{c}0.06 \\
(0.11)\end{array}$ & $\begin{array}{l}-0.03 \\
(0.05)\end{array}$ & $\begin{array}{c}0.04 \\
(0.18)\end{array}$ \\
\hline NET & $\begin{array}{c}0.39 \\
(0.19)^{* *}\end{array}$ & $\begin{array}{c}0.23 \\
(0.09)^{* * *}\end{array}$ & $\begin{array}{c}0.16 \\
(0.10)\end{array}$ & $\begin{array}{c}0.07 \\
(0.04)^{*}\end{array}$ & $\begin{array}{c}0.40 \\
(0.16)^{* * *}\end{array}$ \\
\hline CHAIN & $\begin{array}{c}-0.26 \\
(0.11)^{* *}\end{array}$ & $\begin{array}{c}-0.13 \\
(0.06)^{* *}\end{array}$ & $\begin{array}{c}-0.14 \\
(0.06)^{* *}\end{array}$ & $\begin{array}{c}0.01 \\
(0.03)\end{array}$ & $\begin{array}{c}-0.22 \\
(0.09)^{* *}\end{array}$ \\
\hline AUTONOMOUS & $\begin{array}{l}-0.02 \\
(0.17)\end{array}$ & $\begin{array}{l}-0.01 \\
(0.08)\end{array}$ & $\begin{array}{l}-0.01 \\
(0.09)\end{array}$ & $\begin{array}{c}0.00 \\
(0.04)\end{array}$ & $\begin{array}{c}-0.02 \\
(0.14)\end{array}$ \\
\hline PROJECT & $\begin{array}{l}-0.28 \\
(0.18)\end{array}$ & $\begin{array}{c}-0.15 \\
(0.09)^{*}\end{array}$ & $\begin{array}{l}-0.13 \\
(0.09)\end{array}$ & $\begin{array}{l}-0.02 \\
(0.04)\end{array}$ & $\begin{array}{l}-0.24 \\
(0.15)\end{array}$ \\
\hline ROTATION & $\begin{array}{l}-0.13 \\
(0.16)\end{array}$ & $\begin{array}{l}-0.06 \\
(0.08)\end{array}$ & $\begin{array}{l}-0.07 \\
(0.08)\end{array}$ & $\begin{array}{c}0.00 \\
(0.04)\end{array}$ & $\begin{array}{l}-0.11 \\
(0.13)\end{array}$ \\
\hline QUALITY & $\begin{array}{l}-0.08 \\
(0.17)\end{array}$ & $\begin{array}{l}-0.08 \\
(0.08)\end{array}$ & $\begin{array}{c}0.00 \\
(0.09)\end{array}$ & $\begin{array}{c}-0.07 \\
(0.04)^{* *}\end{array}$ & $\begin{array}{l}-0.09 \\
(0.14)\end{array}$ \\
\hline HIERARCHY & $\begin{array}{c}0.05 \\
(0.11)\end{array}$ & $\begin{array}{c}0.02 \\
(0.05)\end{array}$ & $\begin{array}{c}0.02 \\
(0.06)\end{array}$ & $\begin{array}{c}0.00 \\
(0.02)\end{array}$ & $\begin{array}{c}0.01 \\
(0.09)\end{array}$ \\
\hline JUST TIME & $\begin{array}{c}0.08 \\
(0.09)\end{array}$ & $\begin{array}{c}0.04 \\
(0.04)\end{array}$ & $\begin{array}{c}0.04 \\
(0.05)\end{array}$ & $\begin{array}{c}0.00 \\
(0.02)\end{array}$ & $\begin{array}{c}0.06 \\
(0.07)\end{array}$ \\
\hline Variation & $\begin{array}{c}0.01 \\
(0.15)\end{array}$ & $\begin{array}{l}-0.01 \\
(0.07)\end{array}$ & $\begin{array}{c}0.02 \\
(0.08)\end{array}$ & $\begin{array}{l}-0.03 \\
(0.03)\end{array}$ & $\begin{array}{c}-0.01 \\
(0.12)\end{array}$ \\
\hline Tech. change & $\begin{array}{l}-0.35 \\
(0.19)^{*}\end{array}$ & $\begin{array}{c}-0.17 \\
(0.10)^{*}\end{array}$ & $\begin{array}{c}-0.18 \\
(0.10)^{*}\end{array}$ & $\begin{array}{c}0.01 \\
(0.04)\end{array}$ & $\begin{array}{c}-0.32 \\
(0.16)^{* *}\end{array}$ \\
\hline Org. change & $\begin{array}{l}-0.10 \\
(0.15)\end{array}$ & $\begin{array}{l}-0.03 \\
(0.07)\end{array}$ & $\begin{array}{l}-0.07 \\
(0.08)\end{array}$ & $\begin{array}{c}0.04 \\
(0.03)\end{array}$ & $\begin{array}{l}-0.06 \\
(0.13)\end{array}$ \\
\hline Increasing & $\begin{array}{l}-0.18 \\
(0.22)\end{array}$ & $\begin{array}{l}-0.03 \\
(0.11)\end{array}$ & $\begin{array}{l}-0.15 \\
(0.12)\end{array}$ & $\begin{array}{c}0.12 \\
(0.05)^{* *}\end{array}$ & $\begin{array}{l}-0.15 \\
(0.19)\end{array}$ \\
\hline Decreasing & $\begin{array}{l}-0.22 \\
(0.22)\end{array}$ & $\begin{array}{l}-0.12 \\
(0.11)\end{array}$ & $\begin{array}{l}-0.10 \\
(0.12)\end{array}$ & $\begin{array}{l}-0.02 \\
(0.05)\end{array}$ & $\begin{array}{l}-0.18 \\
(0.18)\end{array}$ \\
\hline Hours & $\begin{array}{l}-0.06 \\
(0.21)\end{array}$ & $\begin{array}{l}-0.01 \\
(0.10)\end{array}$ & $\begin{array}{l}-0.05 \\
(0.11)\end{array}$ & $\begin{array}{c}0.04 \\
(0.05)\end{array}$ & $\begin{array}{l}-0.03 \\
(0.17)\end{array}$ \\
\hline P. Employees & $\begin{array}{c}2.91 \\
(0.50)^{* * *}\end{array}$ & $\begin{array}{c}1.49 \\
(0.25)^{* * *}\end{array}$ & $\begin{array}{c}1.42 \\
(0.26)^{* * *}\end{array}$ & $\begin{array}{c}0.07 \\
(0.11)\end{array}$ & $\begin{array}{c}2.44 \\
(0.42)^{* * *}\end{array}$ \\
\hline P. Technicians & $\begin{array}{c}0.99 \\
(0.61)\end{array}$ & $\begin{array}{c}0.50 \\
(0.30)\end{array}$ & $\begin{array}{c}0.49 \\
(0.33)\end{array}$ & $\begin{array}{c}0.01 \\
(0.14)\end{array}$ & $\begin{array}{c}0.61 \\
(0.52)\end{array}$ \\
\hline P. Managers & $\begin{array}{c}0.21 \\
(0.80)\end{array}$ & $\begin{array}{l}-0.23 \\
(0.40)\end{array}$ & $\begin{array}{c}0.45 \\
(0.42)\end{array}$ & $\begin{array}{c}-0.68 \\
(0.18)^{* * *}\end{array}$ & $\begin{array}{l}-0.28 \\
(0.68)\end{array}$ \\
\hline P. Women & $\begin{array}{c}0.57 \\
(0.34)^{*}\end{array}$ & $\begin{array}{c}0.21 \\
(0.17)\end{array}$ & $\begin{array}{c}0.37 \\
(0.18)^{* *}\end{array}$ & $\begin{array}{c}-0.16 \\
(0.08)^{* *}\end{array}$ & $\begin{array}{c}0.37 \\
(0.29)\end{array}$ \\
\hline P. Contract & $\begin{array}{c}1.12 \\
(0.68)^{*}\end{array}$ & $\begin{array}{c}0.55 \\
(0.34)^{*}\end{array}$ & $\begin{array}{c}0.57 \\
(0.36)\end{array}$ & $\begin{array}{l}-0.01 \\
(0.15)\end{array}$ & $\begin{array}{c}1.02 \\
(0.57)^{*}\end{array}$ \\
\hline Constant & $\begin{array}{c}0.15 \\
(0.30)\end{array}$ & $\begin{array}{c}0.13 \\
(0.15)\end{array}$ & $\begin{array}{c}0.03 \\
(0.16)\end{array}$ & $\begin{array}{c}0.10 \\
(0.07)\end{array}$ & $\begin{array}{c}0.26 \\
(0.25)\end{array}$ \\
\hline Sectors $(10)$ & YES & YES & YES & YES & YES \\
\hline Obs. & 1079 & 1076 & 1076 & 1076 & 1076 \\
\hline$R^{2}$ & 0.09 & 0.08 & 0.09 & 0.05 & 0.09 \\
\hline
\end{tabular}

() Standard errors.

For Sectors we write in parentheses the number of economic sectors included in the regression.

*Significant at $10 \% .{ }^{*}$ Significant at $5 \%{ }^{* * *}$ Significant at $1 \%$.

Source: REPONSE, DMMO and EMMO surveys. 


\section{OLS estimations with incremental organization}

Table 17: Effect of additive clusters on the managers' labor flows, entries, exits, net flows and churning flows. French establishments 1998.

\begin{tabular}{|c|c|c|c|c|c|}
\hline MANAGERS & LABOR FLOWS & ENTRIES & EXITS & NET FLOWS & CHURNING \\
\hline TECHNOLOGY & $\begin{array}{l}-0.66 \\
(0.96)\end{array}$ & $\begin{array}{l}-0.33 \\
(0.48)\end{array}$ & $\begin{array}{l}-0.33 \\
(0.48)\end{array}$ & $\begin{array}{c}0.00 \\
(0.01)\end{array}$ & $\begin{array}{l}-0.67 \\
(0.96)\end{array}$ \\
\hline CHAIN & $\begin{array}{l}-0.47 \\
(0.92)\end{array}$ & $\begin{array}{c}-0.22 \\
(0.46)\end{array}$ & $\begin{array}{l}-0.24 \\
(0.46)\end{array}$ & $\begin{array}{c}0.02 \\
(0.01)^{*}\end{array}$ & $\begin{array}{l}-0.45 \\
(0.92)\end{array}$ \\
\hline TEAMWORK & $\begin{array}{c}1.82 \\
(0.61)^{* * *}\end{array}$ & $\begin{array}{c}0.90 \\
(0.31)^{* * *}\end{array}$ & $\begin{array}{c}0.92 \\
(0.31)^{* * *}\end{array}$ & $\begin{array}{c}-0.02 \\
(0.01)^{* *}\end{array}$ & $\begin{array}{c}1.81 \\
(0.61)^{* * *}\end{array}$ \\
\hline FLEXIBILITY & $\begin{array}{c}0.46 \\
(0.62)\end{array}$ & $\begin{array}{c}0.24 \\
(0.31)\end{array}$ & $\begin{array}{c}0.24 \\
(0.31)\end{array}$ & $\begin{array}{c}0.00 \\
(0.01)\end{array}$ & $\begin{array}{c}0.47 \\
(0.62)\end{array}$ \\
\hline QUALITY & $\begin{array}{c}-2.50 \\
(1.35)^{*}\end{array}$ & $\begin{array}{c}-1.26 \\
(0.68)^{*}\end{array}$ & $\begin{array}{c}-1.28 \\
(0.68)^{*}\end{array}$ & $\begin{array}{c}0.02 \\
(0.02)\end{array}$ & $\begin{array}{c}-2.51 \\
(1.35)^{*}\end{array}$ \\
\hline Variation & $\begin{array}{c}0.86 \\
(1.22)\end{array}$ & $\begin{array}{c}0.43 \\
(0.61)\end{array}$ & $\begin{array}{c}0.44 \\
(0.61)\end{array}$ & $\begin{array}{l}-0.01 \\
(0.01)\end{array}$ & $\begin{array}{c}0.87 \\
(1.22)\end{array}$ \\
\hline Tech. change & $\begin{array}{l}-1.28 \\
(1.53)\end{array}$ & $\begin{array}{l}-0.61 \\
(0.77)\end{array}$ & $\begin{array}{l}-0.63 \\
(0.77)\end{array}$ & $\begin{array}{c}0.02 \\
(0.02)\end{array}$ & $\begin{array}{l}-1.25 \\
(1.53)\end{array}$ \\
\hline Org. change & $\begin{array}{c}1.04 \\
(1.25)\end{array}$ & $\begin{array}{c}0.51 \\
(0.63)\end{array}$ & $\begin{array}{c}0.52 \\
(0.63)\end{array}$ & $\begin{array}{l}-0.01 \\
(0.01)\end{array}$ & $\begin{array}{c}1.01 \\
(1.26)\end{array}$ \\
\hline Increasing & $\begin{array}{l}-0.38 \\
(1.77)\end{array}$ & $\begin{array}{l}-0.18 \\
(0.89)\end{array}$ & $\begin{array}{l}-0.21 \\
(0.89)\end{array}$ & $\begin{array}{c}0.03 \\
(0.02)\end{array}$ & $\begin{array}{l}-0.38 \\
(1.77)\end{array}$ \\
\hline Decreasing & $\begin{array}{c}5.02 \\
(1.85)^{* * *}\end{array}$ & $\begin{array}{c}2.52 \\
(0.93)^{* * *}\end{array}$ & $\begin{array}{c}2.51 \\
(0.93)^{* * *}\end{array}$ & $\begin{array}{c}0.01 \\
(0.02)\end{array}$ & $\begin{array}{c}5.02 \\
(1.85)^{* * *}\end{array}$ \\
\hline Hours & $\begin{array}{c}3.96 \\
(1.75)^{* *}\end{array}$ & $\begin{array}{c}2.01 \\
(0.88)^{* *}\end{array}$ & $\begin{array}{c}2.00 \\
(0.88)^{* *}\end{array}$ & $\begin{array}{c}0.02 \\
(0.02)\end{array}$ & $\begin{array}{c}4.03 \\
(1.76)^{* *}\end{array}$ \\
\hline P. Employees & $\begin{array}{c}4.95 \\
(2.95)^{*}\end{array}$ & $\begin{array}{c}2.47 \\
(1.48)^{*}\end{array}$ & $\begin{array}{c}2.54 \\
(1.48)^{*}\end{array}$ & $\begin{array}{c}-0.07 \\
(0.03)^{* *}\end{array}$ & $\begin{array}{c}4.94 \\
(2.96)^{*}\end{array}$ \\
\hline P. Technicians & $\begin{array}{c}-0.52 \\
(4.44)\end{array}$ & $\begin{array}{l}-0.24 \\
(2.22)\end{array}$ & $\begin{array}{c}-0.23 \\
(2.23)\end{array}$ & $\begin{array}{l}-0.01 \\
(0.05)\end{array}$ & $\begin{array}{c}-0.47 \\
(4.45)\end{array}$ \\
\hline P. Managers & $\begin{array}{l}-3.00 \\
(5.14)\end{array}$ & $\begin{array}{l}-1.42 \\
(2.57)\end{array}$ & $\begin{array}{l}-1.58 \\
(2.58)\end{array}$ & $\begin{array}{c}0.16 \\
(0.06)^{* * *}\end{array}$ & $\begin{array}{l}-2.81 \\
(5.15)\end{array}$ \\
\hline P. Women & $\begin{array}{c}0.26 \\
(2.73)\end{array}$ & $\begin{array}{c}0.11 \\
(1.37)\end{array}$ & $\begin{array}{c}0.06 \\
(1.38)\end{array}$ & $\begin{array}{c}0.05 \\
(0.03)^{*}\end{array}$ & $\begin{array}{c}0.17 \\
(2.75)\end{array}$ \\
\hline P. Contract & $\begin{array}{l}-6.46 \\
(5.58)\end{array}$ & $\begin{array}{l}-3.17 \\
(2.79)\end{array}$ & $\begin{array}{l}-3.30 \\
(2.80)\end{array}$ & $\begin{array}{c}0.14 \\
(0.06)^{* *}\end{array}$ & $\begin{array}{l}-6.38 \\
(5.58)\end{array}$ \\
\hline Constant & $\begin{array}{l}-2.38 \\
(2.43)\end{array}$ & $\begin{array}{l}-1.24 \\
(1.22)\end{array}$ & $\begin{array}{l}-1.17 \\
(1.22)\end{array}$ & $\begin{array}{c}-0.07 \\
(0.03)^{* * *}\end{array}$ & $\begin{array}{l}-2.54 \\
(2.43)\end{array}$ \\
\hline Sectors (10) & YES & YES & YES & YES & YES \\
\hline $\begin{array}{l}\text { Obs. } \\
R^{2}\end{array}$ & $\begin{array}{c}1388 \\
0.04 \\
\end{array}$ & $\begin{array}{c}1384 \\
0.04 \\
\end{array}$ & $\begin{array}{l}1384 \\
0.04 \\
\end{array}$ & $\begin{array}{l}1384 \\
0.02 \\
\end{array}$ & $\begin{array}{l}1384 \\
0.04 \\
\end{array}$ \\
\hline
\end{tabular}

() Standard errors.

For Sectors we write in parentheses the number of economic sectors included in the regression.

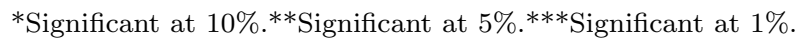

Source: REPONSE, DMMO and EMMO surveys. 
Table 18: Effect of additive clusters on the intermediary professionals' labor flows, entries, exits, net flows and churning flows. French establishments 1998.

\begin{tabular}{|c|c|c|c|c|c|}
\hline INTERMED. PROF. & LABOR FLOWS & ENTRIES & EXITS & NET FLOWS & CHURNING \\
\hline \multirow[t]{2}{*}{ TECHNOLOGY } & 0.09 & 0.05 & 0.04 & 0.01 & 0.09 \\
\hline & $(0.13)$ & $(0.07)$ & $(0.06)$ & $(0.02)$ & $(0.12)$ \\
\hline \multirow[t]{2}{*}{ CHAIN } & 0.05 & 0.04 & 0.01 & 0.02 & 0.08 \\
\hline & $(0.12)$ & $(0.06)$ & $(0.06)$ & $(0.02)$ & $(0.12)$ \\
\hline \multirow[t]{2}{*}{ TEAMWORK } & -0.15 & -0.08 & -0.07 & -0.01 & -0.15 \\
\hline & $(0.08)^{*}$ & $(0.04)^{* *}$ & $(0.04)^{*}$ & $(0.01)$ & $(0.08) *$ \\
\hline \multirow[t]{2}{*}{ FLEXIBILITY } & 0.01 & 0.01 & 0.01 & 0.00 & 0.01 \\
\hline & $(0.08)$ & $(0.04)$ & $(0.04)$ & $(0.01)$ & $(0.08)$ \\
\hline \multirow[t]{2}{*}{ QUALITY } & 0.17 & 0.05 & 0.11 & -0.06 & 0.17 \\
\hline & $(0.18)$ & $(0.09)$ & $(0.09)$ & $(0.03)^{* *}$ & $(0.17)$ \\
\hline \multirow[t]{2}{*}{ Variation } & 0.13 & 0.03 & 0.09 & -0.06 & 0.10 \\
\hline & $(0.16)$ & $(0.08)$ & $(0.08)$ & $(0.03)^{* *}$ & $(0.16)$ \\
\hline \multirow[t]{2}{*}{ Tech. change } & 0.13 & 0.06 & 0.08 & -0.01 & 0.17 \\
\hline & $(0.20)$ & $(0.11)$ & $(0.10)$ & $(0.03)$ & $(0.20)$ \\
\hline \multirow[t]{2}{*}{ Org. change } & 0.01 & 0.00 & 0.01 & -0.01 & -0.06 \\
\hline & $(0.17)$ & $(0.09)$ & $(0.08)$ & $(0.03)$ & $(0.16)$ \\
\hline \multirow[t]{2}{*}{ Increasing } & -0.22 & -0.07 & -0.15 & 0.08 & -0.18 \\
\hline & $(0.24)$ & $(0.12)$ & $(0.12)$ & $(0.04)^{* *}$ & $(0.23)$ \\
\hline \multirow[t]{2}{*}{ Decreasing } & 0.42 & 0.20 & 0.22 & -0.03 & 0.45 \\
\hline & $(0.25)^{*}$ & $(0.13)$ & $(0.12)^{*}$ & $(0.04)$ & $(0.24)^{*}$ \\
\hline \multirow[t]{2}{*}{ Hours } & 0.24 & 0.13 & 0.12 & 0.01 & 0.21 \\
\hline & $(0.23)$ & $(0.12)$ & $(0.12)$ & $(0.04)$ & $(0.22)$ \\
\hline \multirow[t]{2}{*}{ P. Employees } & 0.72 & 0.38 & 0.34 & 0.04 & 0.56 \\
\hline & $(0.39)^{*}$ & $(0.20)^{*}$ & $(0.20)^{*}$ & $(0.06)$ & $(0.38)$ \\
\hline \multirow[t]{2}{*}{ P. Technicians } & -3.18 & -1.61 & -1.57 & -0.05 & -2.63 \\
\hline & $(0.59)^{* * *}$ & $(0.31)^{* * *}$ & $(0.30)^{* * *}$ & $(0.10)$ & $(0.57)^{* * *}$ \\
\hline \multirow[t]{2}{*}{ P. Managers } & 3.10 & 1.67 & 1.44 & 0.23 & 2.73 \\
\hline & $(0.68)^{* * *}$ & $(0.35)^{* * *}$ & $(0.34)^{* * *}$ & $(0.11)^{* *}$ & $(0.66)^{* * *}$ \\
\hline \multirow[t]{2}{*}{ P. Women } & 0.35 & 0.13 & 0.22 & -0.09 & 0.37 \\
\hline & $(0.36)$ & $(0.19)$ & $(0.18)$ & $(0.06)$ & $(0.35)$ \\
\hline \multirow[t]{2}{*}{ P. Contract } & 0.77 & 0.46 & 0.30 & 0.16 & 0.70 \\
\hline & $(0.74)$ & $(0.38)$ & $(0.37)$ & $(0.12)$ & $(0.71)$ \\
\hline \multirow[t]{2}{*}{ Constant } & 0.10 & 0.07 & 0.03 & 0.04 & -0.08 \\
\hline & $(0.33)$ & $(0.17)$ & $(0.16)$ & $(0.05)$ & $(0.31)$ \\
\hline Sectors (10) & YES & YES & YES & YES & YES \\
\hline Obs. & 1374 & 1371 & 1371 & 1371 & 1371 \\
\hline$R^{2}$ & 0.15 & 0.15 & 0.14 & 0.02 & 0.14 \\
\hline
\end{tabular}

() Standard errors.

For Sectors we write in parentheses the number of economic sectors included in the regression.

*Significant at $10 \%{ }^{* *}$ Significant at $5 \%$.***Significant at $1 \%$.

Source: REPONSE, DMMO and EMMO surveys. 
Table 19: Effect of additive clusters on the manual workers' labor flows, entries, exits, net flows and churning flows. French establishments 1998.

\begin{tabular}{|l|ccccc|}
\hline & & & & & \\
MANUAL WORK. & LABOR FLOWS & ENTRIES & EXITS & NET FLOWS & CHURNING \\
\hline TECHNOLOGY & & & & & \\
& 0.24 & 0.13 & 0.11 & 0.02 & 0.23 \\
CHAIN & $(0.12)^{* *}$ & $(0.06)^{* *}$ & $(0.06)^{*}$ & $(0.03)$ & $(0.10)^{* *}$ \\
& -0.25 & -0.12 & -0.13 & 0.01 & -0.21 \\
TEAMWORK & $(0.11)^{* *}$ & $(0.05)^{* *}$ & $(0.06)^{* *}$ & $(0.03)$ & $(0.09)^{* *}$ \\
FLEXIBILITY & -0.04 & -0.02 & -0.02 & 0.00 & -0.05 \\
& $(0.07)$ & $(0.04)$ & $(0.04)$ & $(0.02)$ & $(0.06)$ \\
QUALITY & 0.03 & 0.02 & 0.01 & 0.00 & 0.02 \\
& $(0.07)$ & $(0.04)$ & $(0.04)$ & $(0.02)$ & $(0.06)$ \\
Variation & -0.08 & -0.07 & 0.00 & -0.07 & -0.08 \\
& $(0.17)$ & $(0.08)$ & $(0.09)$ & $(0.04)^{*}$ & $(0.14)$ \\
Tech. change & 0.01 & -0.01 & 0.02 & -0.03 & -0.01 \\
Org. change & $(0.15)$ & $(0.07)$ & $(0.08)$ & $(0.03)$ & $(0.12)$ \\
& -0.35 & -0.17 & -0.18 & 0.01 & -0.32 \\
Increasing & $(0.19)^{*}$ & $(0.10)^{*}$ & $(0.10)^{*}$ & $(0.04)$ & $(0.16)^{* *}$ \\
& -0.09 & -0.02 & -0.07 & 0.04 & -0.06 \\
Decreasing & $(0.15)$ & $(0.07)$ & $(0.08)$ & $(0.03)$ & $(0.13)$ \\
& -0.17 & -0.02 & -0.14 & 0.12 & -0.14 \\
Hours & $(0.22)$ & $(0.11)$ & $(0.12)$ & $(0.05)^{* *}$ & $(0.19)$ \\
P. Employees & -0.20 & -0.11 & -0.09 & -0.03 & -0.17 \\
P. Technicians & $(0.22)$ & $(0.11)$ & $(0.11)$ & $(0.05)$ & $(0.18)$ \\
& -0.04 & 0.00 & -0.04 & 0.04 & -0.02 \\
P. Managers & $(0.20)$ & $(0.10)$ & $(0.11)$ & $(0.05)$ & $(0.17)$ \\
P. Women & 2.85 & 1.45 & 1.39 & 0.06 & 2.39 \\
P. Contract & $(0.49)^{* * *}$ & $(0.25)^{* * *}$ & $(0.26)^{* * *}$ & $(0.11)$ & $(0.42)^{* * *}$ \\
Constant & 0.85 & 0.41 & 0.43 & -0.02 & 0.48 \\
Sectors $(10)$ & $(0.61)$ & $(0.30)$ & $(0.32)$ & $(0.14)$ & $(0.51)$ \\
\hline Obs. & 0.13 & -0.28 & 0.42 & -0.70 & -0.37 \\
$R^{2}$ & $(0.79)$ & $(0.39)$ & $(0.42)$ & $(0.18)^{* * *}$ & $(0.67)$ \\
& 0.55 & 0.20 & 0.37 & -0.16 & 0.35 \\
& $(0.34)^{*}$ & $(0.17)$ & $(0.18)^{* *}$ & $(0.08)^{* *}$ & $(0.29)$ \\
& 1.09 & 0.53 & 0.55 & -0.02 & 1.00 \\
& $(0.68)$ & $(0.34)$ & $(0.36)$ & $(0.15)$ & $(0.57)^{*}$ \\
& 0.21 & 0.16 & 0.06 & 0.11 & 0.31 \\
& $(0.29)$ & $(0.14)$ & $(0.15)$ & $(0.07)$ & $(0.25)$ \\
& YES & YES & YES & YES & YES \\
\hline & 1079 & 1076 & 1076 & 1076 & 1076 \\
& 0.09 & 0.08 & 0.09 & 0.06 & 0.09 \\
\hline
\end{tabular}

() Standard errors.

For Sectors we write in parentheses the number of economic sectors included in the regression.

*Significant at $10 \% .{ }^{* *}$ Significant at $5 \% . * *$ Significant at $1 \%$.

Source: REPONSE, DMMO and EMMO surveys. 
OLS estimations with multiplicative clusters 
Table 20: Effect of multiplicative clusters on the managers' labor flows, entries, exits, net flows and churning flows. French establishments 1998.

\begin{tabular}{|c|c|c|c|c|c|}
\hline MANAGERS & LABOR FLOWS & ENTRIES & EXITS & NET FLOWS & CHURNING \\
\hline COMPUTER & $\begin{array}{l}-1.08 \\
(1.87)\end{array}$ & $\begin{array}{l}-0.52 \\
(0.94)\end{array}$ & $\begin{array}{l}-0.52 \\
(0.94)\end{array}$ & $\begin{array}{c}0.00 \\
(0.02)\end{array}$ & $\begin{array}{l}-1.07 \\
(1.87)\end{array}$ \\
\hline NET & $\begin{array}{l}-0.44 \\
(1.53)\end{array}$ & $\begin{array}{l}-0.23 \\
(0.77)\end{array}$ & $\begin{array}{l}-0.23 \\
(0.77)\end{array}$ & $\begin{array}{c}0.00 \\
(0.02)\end{array}$ & $\begin{array}{l}-0.46 \\
(1.53)\end{array}$ \\
\hline CHAIN & $\begin{array}{l}-0.59 \\
(0.92)\end{array}$ & $\begin{array}{l}-0.29 \\
(0.46)\end{array}$ & $\begin{array}{l}-0.31 \\
(0.46)\end{array}$ & $\begin{array}{c}0.02 \\
(0.01)^{*}\end{array}$ & $\begin{array}{l}-0.57 \\
(0.92)\end{array}$ \\
\hline AUTONOMOUS & $\begin{array}{l}-0.11 \\
(1.67)\end{array}$ & $\begin{array}{l}-0.07 \\
(0.84)\end{array}$ & $\begin{array}{l}-0.03 \\
(0.84)\end{array}$ & $\begin{array}{c}-0.04 \\
(0.02)^{* *}\end{array}$ & $\begin{array}{l}-0.12 \\
(1.68)\end{array}$ \\
\hline PROJECT & $\begin{array}{c}0.70 \\
(1.62)\end{array}$ & $\begin{array}{c}0.35 \\
(0.81)\end{array}$ & $\begin{array}{c}0.34 \\
(0.81)\end{array}$ & $\begin{array}{c}0.00 \\
(0.02)\end{array}$ & $\begin{array}{c}0.69 \\
(1.62)\end{array}$ \\
\hline ROTATION & $\begin{array}{l}-1.40 \\
(1.53)\end{array}$ & $\begin{array}{l}-0.69 \\
(0.76)\end{array}$ & $\begin{array}{l}-0.69 \\
(0.77)\end{array}$ & $\begin{array}{c}0.00 \\
(0.02)\end{array}$ & $\begin{array}{l}-1.41 \\
(1.53)\end{array}$ \\
\hline QUALITY & $\begin{array}{c}-2.47 \\
(1.35)^{*}\end{array}$ & $\begin{array}{c}-1.24 \\
(0.67)^{*}\end{array}$ & $\begin{array}{l}-1.26 \\
(0.68)^{*}\end{array}$ & $\begin{array}{c}0.02 \\
(0.02)\end{array}$ & $\begin{array}{l}-2.47 \\
(1.35)^{*}\end{array}$ \\
\hline HIERARCHY & $\begin{array}{c}0.43 \\
(0.88)\end{array}$ & $\begin{array}{c}0.20 \\
(0.44)\end{array}$ & $\begin{array}{c}0.23 \\
(0.44)\end{array}$ & $\begin{array}{c}-0.02 \\
(0.01)^{* *}\end{array}$ & $\begin{array}{c}0.41 \\
(0.88)\end{array}$ \\
\hline JUST TIME & $\begin{array}{c}0.97 \\
(0.73)\end{array}$ & $\begin{array}{c}0.50 \\
(0.37)\end{array}$ & $\begin{array}{c}0.49 \\
(0.37)\end{array}$ & $\begin{array}{c}0.00 \\
(0.01)\end{array}$ & $\begin{array}{c}0.99 \\
(0.73)\end{array}$ \\
\hline TEAMWORK* & $\begin{array}{c}59.47 \\
(1.79)^{* * *}\end{array}$ & $\begin{array}{c}2.98 \\
(0.89)^{* * *}\end{array}$ & $\begin{array}{c}2.96 \\
(0.90)^{* * *}\end{array}$ & $\begin{array}{c}0.01 \\
(0.02)\end{array}$ & $\begin{array}{c}5.96 \\
(1.79)^{* * *}\end{array}$ \\
\hline ICT FLEXIBILITY & $\begin{array}{c}-0.36 \\
(3.16)\end{array}$ & $\begin{array}{c}-0.20 \\
(1.58)\end{array}$ & $\begin{array}{c}-0.20 \\
(1.59)\end{array}$ & $\begin{array}{c}0.00 \\
(0.04)\end{array}$ & $\begin{array}{l}-0.35 \\
(3.17)\end{array}$ \\
\hline Variation & $\begin{array}{c}0.83 \\
(1.21)\end{array}$ & $\begin{array}{c}0.42 \\
(0.61)\end{array}$ & $\begin{array}{c}0.43 \\
(0.61)\end{array}$ & $\begin{array}{l}-0.01 \\
(0.01)\end{array}$ & $\begin{array}{c}0.85 \\
(1.22)\end{array}$ \\
\hline Tech. change & $\begin{array}{l}-1.61 \\
(1.53)\end{array}$ & $\begin{array}{l}-0.78 \\
(0.77)\end{array}$ & $\begin{array}{l}-0.80 \\
(0.77)\end{array}$ & $\begin{array}{c}0.02 \\
(0.02)\end{array}$ & $\begin{array}{l}-1.58 \\
(1.53)\end{array}$ \\
\hline Org. change & $\begin{array}{c}1.15 \\
(1.26)\end{array}$ & $\begin{array}{c}0.57 \\
(0.63)\end{array}$ & $\begin{array}{c}0.58 \\
(0.63)\end{array}$ & $\begin{array}{l}-0.01 \\
(0.01)\end{array}$ & $\begin{array}{c}1.12 \\
(1.26)\end{array}$ \\
\hline Increasing & $\begin{array}{l}-0.60 \\
(1.77)\end{array}$ & $\begin{array}{l}-0.29 \\
(0.88)\end{array}$ & $\begin{array}{l}-0.32 \\
(0.89)\end{array}$ & $\begin{array}{c}0.03 \\
(0.02)\end{array}$ & $\begin{array}{l}-0.60 \\
(1.77)\end{array}$ \\
\hline Decreasing & $\begin{array}{c}4.96 \\
(1.85)^{* * *}\end{array}$ & $\begin{array}{c}2.48 \\
(0.92)^{* * *}\end{array}$ & $\begin{array}{c}2.47 \\
(0.93)^{* * *}\end{array}$ & $\begin{array}{c}0.01 \\
(0.02)\end{array}$ & $\begin{array}{c}4.94 \\
(1.85)^{* * *}\end{array}$ \\
\hline Hours & $\begin{array}{c}4.48 \\
(1.76)^{* * *}\end{array}$ & $\begin{array}{c}2.28 \\
(0.88)^{* * *}\end{array}$ & $\begin{array}{c}2.26 \\
(0.88)^{* * *}\end{array}$ & $\begin{array}{c}0.02 \\
(0.02)\end{array}$ & $\begin{array}{c}4.56 \\
(1.76)^{* * *}\end{array}$ \\
\hline P. Employees & $\begin{array}{c}4.83 \\
(2.95)^{*}\end{array}$ & $\begin{array}{c}2.41 \\
(1.48)\end{array}$ & $\begin{array}{c}2.48 \\
(1.48)^{*}\end{array}$ & $\begin{array}{c}-0.07 \\
(0.03)^{* *}\end{array}$ & $\begin{array}{c}4.82 \\
(2.96)\end{array}$ \\
\hline P. Technicians & $\begin{array}{l}-0.18 \\
(4.47)\end{array}$ & $\begin{array}{l}-0.08 \\
(2.24)\end{array}$ & $\begin{array}{l}-0.07 \\
(2.24)\end{array}$ & $\begin{array}{l}-0.02 \\
(0.05)\end{array}$ & $\begin{array}{l}-0.14 \\
(4.47)\end{array}$ \\
\hline P. Managers & $\begin{array}{l}-2.86 \\
(5.28)\end{array}$ & $\begin{array}{l}-1.36 \\
(2.64)\end{array}$ & $\begin{array}{l}-1.50 \\
(2.65)\end{array}$ & $\begin{array}{c}0.14 \\
(0.06)^{* *}\end{array}$ & $\begin{array}{l}-2.67 \\
(5.28)\end{array}$ \\
\hline P. Women & $\begin{array}{c}0.77 \\
(2.73)\end{array}$ & $\begin{array}{c}0.37 \\
(1.37)\end{array}$ & $\begin{array}{c}0.32 \\
(1.37)\end{array}$ & $\begin{array}{c}0.05 \\
(0.03)^{*}\end{array}$ & $\begin{array}{c}0.69 \\
(2.74)\end{array}$ \\
\hline P. Contract & $\begin{array}{l}-7.03 \\
(5.57)\end{array}$ & $\begin{array}{l}-3.45 \\
(2.78)\end{array}$ & $\begin{array}{l}-3.58 \\
(2.79)\end{array}$ & $\begin{array}{c}0.13 \\
(0.06)^{* *}\end{array}$ & $\begin{array}{l}-6.95 \\
(5.57)\end{array}$ \\
\hline Constant & $\begin{array}{l}-0.84 \\
(2.48)\end{array}$ & $\begin{array}{l}-0.46 \\
(1.24)\end{array}$ & $\begin{array}{l}-0.40 \\
(1.25)\end{array}$ & $\begin{array}{c}-0.06 \\
(0.03)^{* *}\end{array}$ & $\begin{array}{l}-0.98 \\
(2.48)\end{array}$ \\
\hline Sectors (10) & YES & YES & YES & YES & YES \\
\hline $\begin{array}{l}\text { Obs. } \\
R^{2}\end{array}$ & $\begin{array}{l}1388 \\
0.05\end{array}$ & $\begin{array}{l}1384 \\
0.05\end{array}$ & $\begin{array}{l}1384 \\
0.05\end{array}$ & $\begin{array}{l}1384 \\
0.02\end{array}$ & $\begin{array}{l}1384 \\
0.05\end{array}$ \\
\hline
\end{tabular}

() Standard errors.

For Sectors we write in parentheses the number of economic sectors included in the regression.

*Significant at $10 \%{ }^{* *}$ Significant at $5 \%{ }^{* * *}$ Significant at $1 \%$.

Source: REPONSE, DMMO and EMMO surveys. 
Table 21: Effect of multiplicative clusters on the intermediary professionals' labor flows, entries, exits, net flows and churning flows. French establishments 1998.

\begin{tabular}{|c|c|c|c|c|c|}
\hline INTERMED. PROF. & LABOR FLOWS & ENTRIES & EXITS & NET FLOWS & CHURNING \\
\hline COMPUTER & 0.18 & 0.12 & $\begin{array}{c}0.07 \\
(0.12)\end{array}$ & 0.05 & $\begin{array}{c}0.15 \\
(0.24)\end{array}$ \\
\hline NET & -0.01 & -0.02 & 0.00 & -0.02 & 0.03 \\
\hline CHAIN & $\begin{array}{c}(0.21) \\
0.06 \\
(0.12)\end{array}$ & $\begin{array}{c}(0.11) \\
0.04 \\
(0.06)\end{array}$ & $\begin{array}{c}(0.10) \\
0.02 \\
(0.06)\end{array}$ & $\begin{array}{c}(0.03) \\
0.02 \\
(0.02)\end{array}$ & $\begin{array}{c}(0.20) \\
0.09 \\
(0.12)\end{array}$ \\
\hline AUTONOMOUS & $\begin{array}{c}0.11 \\
(0.22)\end{array}$ & $\begin{array}{c}0.03 \\
(0.12)\end{array}$ & $\begin{array}{c}0.07 \\
(0.11)\end{array}$ & $\begin{array}{l}-0.04 \\
(0.04)\end{array}$ & $\begin{array}{c}0.09 \\
(0.21)\end{array}$ \\
\hline PROJECT & $\begin{array}{l}-0.31 \\
(0.22)\end{array}$ & $\begin{array}{l}-0.16 \\
(0.11)\end{array}$ & $\begin{array}{l}-0.15 \\
(0.11)\end{array}$ & $\begin{array}{l}-0.01 \\
(0.04)\end{array}$ & $\begin{array}{l}-0.32 \\
(0.21)\end{array}$ \\
\hline ROTATION & $\begin{array}{c}0.14 \\
(0.20)\end{array}$ & $\begin{array}{c}0.07 \\
(0.11)\end{array}$ & $\begin{array}{c}0.06 \\
(0.10)\end{array}$ & $\begin{array}{c}0.01 \\
(0.03)\end{array}$ & $\begin{array}{c}0.12 \\
(0.20)\end{array}$ \\
\hline QUALITY & $\begin{array}{c}0.18 \\
(0.18)\end{array}$ & $\begin{array}{c}0.06 \\
(0.09)\end{array}$ & $\begin{array}{c}0.12 \\
(0.09)\end{array}$ & $\begin{array}{c}-0.06 \\
(0.03)^{* *}\end{array}$ & $\begin{array}{c}0.18 \\
(0.17)\end{array}$ \\
\hline HIERARCHY & $\begin{array}{l}-0.21 \\
(0.12)^{*}\end{array}$ & $\begin{array}{c}-0.11 \\
(0.06)^{*}\end{array}$ & $\begin{array}{l}-0.10 \\
(0.06)\end{array}$ & $\begin{array}{l}-0.01 \\
(0.02)\end{array}$ & $\begin{array}{l}-0.21 \\
(0.11)^{*}\end{array}$ \\
\hline JUST TIME & $\begin{array}{l}-0.04 \\
(0.10)\end{array}$ & $\begin{array}{l}-0.02 \\
(0.05)\end{array}$ & $\begin{array}{l}-0.02 \\
(0.05)\end{array}$ & $\begin{array}{c}0.00 \\
(0.02)\end{array}$ & $\begin{array}{l}-0.04 \\
(0.09)\end{array}$ \\
\hline TEAMWORK* & $\begin{array}{c}0.07 \\
(0.24)\end{array}$ & $\begin{array}{c}0.04 \\
(0.12)\end{array}$ & $\begin{array}{c}0.03 \\
(0.12)\end{array}$ & $\begin{array}{c}0.02 \\
(0.04)\end{array}$ & $\begin{array}{c}0.11 \\
(0.23)\end{array}$ \\
\hline ICT FLEXIBILITY & $\begin{array}{c}0.23 \\
(0.42)\end{array}$ & $\begin{array}{c}0.10 \\
(0.22)\end{array}$ & $\begin{array}{c}0.12 \\
(0.21)\end{array}$ & $\begin{array}{l}-0.02 \\
(0.07)\end{array}$ & $\begin{array}{c}0.10 \\
(0.41)\end{array}$ \\
\hline Variation & $\begin{array}{c}0.13 \\
(0.16)\end{array}$ & $\begin{array}{c}0.03 \\
(0.08)\end{array}$ & $\begin{array}{c}0.09 \\
(0.08)\end{array}$ & $\begin{array}{c}-0.06 \\
(0.03)^{* *}\end{array}$ & $\begin{array}{c}0.10 \\
(0.16)\end{array}$ \\
\hline Tech. change & $\begin{array}{c}0.13 \\
(0.20)\end{array}$ & $\begin{array}{c}0.06 \\
(0.11)\end{array}$ & $\begin{array}{c}0.08 \\
(0.10)\end{array}$ & $\begin{array}{l}-0.02 \\
(0.03)\end{array}$ & $\begin{array}{c}0.16 \\
(0.20)\end{array}$ \\
\hline Org. change & $\begin{array}{c}0.00 \\
(0.17)\end{array}$ & $\begin{array}{l}-0.01 \\
(0.09)\end{array}$ & $\begin{array}{c}0.00 \\
(0.08)\end{array}$ & $\begin{array}{l}-0.01 \\
(0.03)\end{array}$ & $\begin{array}{l}-0.07 \\
(0.16)\end{array}$ \\
\hline Increasing & $\begin{array}{l}-0.23 \\
(0.24)\end{array}$ & $\begin{array}{l}-0.08 \\
(0.12)\end{array}$ & $\begin{array}{l}-0.16 \\
(0.12)\end{array}$ & $\begin{array}{c}0.08 \\
(0.04)^{* *}\end{array}$ & $\begin{array}{l}-0.19 \\
(0.23)\end{array}$ \\
\hline Decreasing & $\begin{array}{c}0.44 \\
(0.25)^{*}\end{array}$ & $\begin{array}{c}0.20 \\
(0.13)\end{array}$ & $\begin{array}{c}0.23 \\
(0.12)^{*}\end{array}$ & $\begin{array}{l}-0.03 \\
(0.04)\end{array}$ & $\begin{array}{c}0.47 \\
(0.24)^{* *}\end{array}$ \\
\hline Hours & $\begin{array}{c}0.26 \\
(0.23)\end{array}$ & $\begin{array}{c}0.14 \\
(0.12)\end{array}$ & $\begin{array}{c}0.13 \\
(0.12)\end{array}$ & $\begin{array}{c}0.01 \\
(0.04)\end{array}$ & $\begin{array}{c}0.23 \\
(0.23)\end{array}$ \\
\hline P. Employees & $\begin{array}{c}0.71 \\
(0.39)^{*}\end{array}$ & $\begin{array}{c}0.37 \\
(0.20)^{*}\end{array}$ & $\begin{array}{c}0.34 \\
(0.20)^{*}\end{array}$ & $\begin{array}{c}0.04 \\
(0.07)\end{array}$ & $\begin{array}{c}0.57 \\
(0.38)\end{array}$ \\
\hline P. Technicians & $\begin{array}{c}-3.22 \\
(0.60)^{* * *}\end{array}$ & $\begin{array}{c}-1.64 \\
(0.31)^{* * *}\end{array}$ & $\begin{array}{c}-1.58 \\
(0.30)^{* * *}\end{array}$ & $\begin{array}{l}-0.06 \\
(0.10)\end{array}$ & $\begin{array}{c}-2.64 \\
(0.58)^{* * *}\end{array}$ \\
\hline P. Managers & $\begin{array}{c}3.30 \\
(0.70)^{* * *}\end{array}$ & $\begin{array}{c}1.76 \\
(0.36)^{* * *}\end{array}$ & $\begin{array}{c}1.55 \\
(0.35)^{* * *}\end{array}$ & $\begin{array}{c}0.21 \\
(0.12)^{*}\end{array}$ & $\begin{array}{c}2.93 \\
(0.68)^{* * *}\end{array}$ \\
\hline P. Women & $\begin{array}{c}0.35 \\
(0.36)\end{array}$ & $\begin{array}{c}0.13 \\
(0.19)\end{array}$ & $\begin{array}{c}0.22 \\
(0.18)\end{array}$ & $\begin{array}{l}-0.09 \\
(0.06)\end{array}$ & $\begin{array}{c}0.38 \\
(0.35)\end{array}$ \\
\hline P. Contract & $\begin{array}{c}0.73 \\
(0.74)\end{array}$ & $\begin{array}{c}0.44 \\
(0.38)\end{array}$ & $\begin{array}{c}0.28 \\
(0.37)\end{array}$ & $\begin{array}{c}0.16 \\
(0.12)\end{array}$ & $\begin{array}{c}0.67 \\
(0.71)\end{array}$ \\
\hline Constant & $\begin{array}{c}0.10 \\
(0.33)\end{array}$ & $\begin{array}{c}0.08 \\
(0.17)\end{array}$ & $\begin{array}{c}0.03 \\
(0.17)\end{array}$ & $\begin{array}{c}0.05 \\
(0.06)\end{array}$ & $\begin{array}{l}-0.08 \\
(0.32)\end{array}$ \\
\hline Sectors (10) & YES & YES & YES & YES & YES \\
\hline Obs. & 1374 & 1371 & 1371 & 1371 & 1371 \\
\hline$R^{2}$ & 0.14 & 0.15 & 0.14 & 0.01 & 0.14 \\
\hline
\end{tabular}

() Standard errors.

For Sectors we write in parentheses the number of economic sectors included in the regression.

*Significant at $10 \%$.**Significant at $5 \%{ }^{* * *}$ Significant at $1 \%$.

Source: REPONSE, DMMO and EMMO surveys. 
Table 22: Effect of multiplicative clusters on the employees' labor flows, entries, exits, net flows and churning flows. French establishments 1998.

\begin{tabular}{|c|c|c|c|c|c|}
\hline EMPLOYEES & LABOR FLOWS & ENTRIES & EXITS & NET FLOWS & CHURNING \\
\hline \multirow[t]{2}{*}{ COMPUTER } & 0.25 & 0.12 & 0.11 & 0.01 & 0.23 \\
\hline & $(0.40)$ & $(0.22)$ & $(0.19)$ & $(0.08)$ & $(0.37)$ \\
\hline \multirow[t]{2}{*}{ NET } & -0.06 & -0.03 & -0.03 & 0.00 & -0.06 \\
\hline & $(0.33)$ & $(0.18)$ & $(0.15)$ & $(0.06)$ & $(0.31)$ \\
\hline \multirow[t]{2}{*}{ CHAIN } & -0.20 & -0.08 & -0.12 & 0.04 & -0.20 \\
\hline & $(0.20)$ & $(0.11)$ & $(0.09)$ & $(0.04)$ & $(0.18)$ \\
\hline \multirow[t]{2}{*}{ AUTONOMOUS } & -0.34 & -0.22 & -0.13 & -0.09 & -0.29 \\
\hline & $(0.36)$ & $(0.20)$ & $(0.17)$ & $(0.07)$ & $(0.33)$ \\
\hline \multirow[t]{2}{*}{ PROJECT } & -0.37 & -0.20 & -0.17 & -0.03 & -0.34 \\
\hline & $(0.35)$ & $(0.19)$ & $(0.16)$ & $(0.07)$ & $(0.32)$ \\
\hline \multirow[t]{2}{*}{ ROTATION } & -0.08 & -0.08 & -0.01 & -0.07 & -0.05 \\
\hline & $(0.32)$ & $(0.18)$ & $(0.15)$ & $(0.06)$ & $(0.30)$ \\
\hline \multirow[t]{2}{*}{ QUALITY } & 0.00 & 0.03 & -0.02 & 0.05 & -0.04 \\
\hline & $(0.29)$ & $(0.16)$ & $(0.13)$ & $(0.05)$ & $(0.27)$ \\
\hline \multirow[t]{2}{*}{ HIERARCHY } & -0.45 & -0.26 & -0.19 & -0.07 & -0.39 \\
\hline & $(0.19)^{*}$ & $(0.10)^{* * *}$ & $(0.09)^{* *}$ & $(0.04)^{*}$ & $(0.18)^{* *}$ \\
\hline \multirow[t]{2}{*}{ JUST TIME } & -0.05 & -0.04 & -0.01 & -0.03 & -0.03 \\
\hline & $(0.16)$ & $(0.09)$ & $(0.07)$ & $(0.03)$ & $(0.15)$ \\
\hline \multirow[t]{2}{*}{ TEAMWORK* } & 0.38 & 0.22 & 0.17 & 0.05 & 0.34 \\
\hline & $(0.38)$ & $(0.21)$ & $(0.18)$ & $(0.07)$ & $(0.36)$ \\
\hline \multirow[t]{2}{*}{ ICT FLEXIBILITY } & 2.28 & 1.23 & 1.06 & 0.17 & 2.16 \\
\hline & $(0.68)^{* * *}$ & $(0.37)^{* * *}$ & $(0.32)^{* * *}$ & $(0.13)$ & $(0.64)^{* * *}$ \\
\hline \multirow[t]{2}{*}{ Variation } & 0.09 & 0.03 & 0.06 & -0.04 & 0.09 \\
\hline & $(0.26)$ & $(0.14)$ & $(0.12)$ & $(0.05)$ & $(0.24)$ \\
\hline \multirow[t]{2}{*}{ Tech. change } & -0.13 & -0.10 & -0.04 & -0.06 & -0.11 \\
\hline & $(0.33)$ & $(0.18)$ & $(0.15)$ & $(0.06)$ & $(0.30)$ \\
\hline \multirow[t]{2}{*}{ Org. change } & 0.40 & 0.25 & 0.14 & 0.11 & 0.30 \\
\hline & $(0.27)$ & $(0.15)^{*}$ & $(0.13)$ & $(0.05)^{* *}$ & $(0.25)$ \\
\hline \multirow[t]{2}{*}{ Increasing } & -0.06 & -0.03 & -0.03 & 0.00 & 0.00 \\
\hline & $(0.38)$ & $(0.21)$ & $(0.18)$ & $(0.07)$ & $(0.35)$ \\
\hline \multirow[t]{2}{*}{ Decreasing } & -0.09 & -0.07 & -0.03 & -0.04 & -0.06 \\
\hline & $(0.39)$ & $(0.22)$ & $(0.18)$ & $(0.07)$ & $(0.37)$ \\
\hline \multirow[t]{2}{*}{ Hours } & -0.25 & -0.13 & -0.12 & -0.01 & -0.21 \\
\hline & $(0.37)$ & $(0.21)$ & $(0.18)$ & $(0.07)$ & $(0.35)$ \\
\hline \multirow[t]{2}{*}{ P. Employees } & -3.94 & -2.14 & -1.81 & -0.33 & -3.50 \\
\hline & $(0.63)^{* * *}$ & $(0.34)^{* * *}$ & $(0.29)^{* * *}$ & $(0.12)^{* * *}$ & $(0.58)^{* * *}$ \\
\hline \multirow[t]{2}{*}{ P. Technicians } & -2.39 & -1.38 & -1.02 & -0.36 & -2.17 \\
\hline & $(0.97)^{* *}$ & $(0.53)^{* * *}$ & $(0.46)^{* *}$ & $(0.18)^{* *}$ & $(0.91)^{* *}$ \\
\hline P. Managers & 0.16 & 0.00 & 0.16 & -0.15 & $\begin{array}{c}0.42 \\
(106)\end{array}$ \\
\hline \multirow[t]{2}{*}{ P. Women } & 1.60 & 0.79 & 0.84 & -0.05 & 1.59 \\
\hline & $(0.58) * * *$ & $(0.32)^{* * *}$ & $(0.27)^{* * *}$ & $(0.11)$ & $(0.54)^{* * *}$ \\
\hline \multirow[t]{2}{*}{ P. Contract } & 2.76 & 1.39 & 1.37 & 0.03 & 2.56 \\
\hline & $(1.18)^{* *}$ & $(0.65)^{* *}$ & $(0.55)^{* *}$ & $(0.22)$ & $(1.10)^{* *}$ \\
\hline Constant & 1.32 & 0.75 & 0.58 & 0.17 & 1.08 \\
\hline & $(0.53)^{* * *}$ & $(0.29)^{* * *}$ & $(0.25)^{* *}$ & $(0.10)^{*}$ & $(0.49)^{* *}$ \\
\hline Sectors (10) & YES & YES & YES & YES & YES \\
\hline Obs. & 1359 & 1355 & 1355 & 1355 & 1355 \\
\hline$R^{2}$ & 0.08 & 0.08 & 0.08 & 0.01 & 0.08 \\
\hline
\end{tabular}

() Standard errors.

For Sectors we write in parentheses the number of economic sectors included in the regression.

${ }^{*}$ Significant at $10 \%{ }^{* *}$ Significant at $5 \% . * * *$ Significant at $1 \%$.

Source: REPONSE, DMMO and EMMO surveys. 